\title{
S-BDS: An Effective Blockchain-based Data Storage Scheme in Zero-Trust loT
}

Jin Wang

School of Computer Science and Mathematics, Fujian University of Technology; School of Computer \&

Communication Engineering, Changsha University of Science \& Technology, China, jinwang@csust.edu.cn

Jiahao Chen

School of Computer Science and Mathematics, Fujian University of Technology, China, chen1014036360@126.com

Neal Xiong*

Department of Computer Science and Mathematics, Sul Ross State University, USA, xiongnaixue@gmail.com

Osama Alfarraj

Computer Science Department, Community College, King Saud University, Saudi Arabia, oalfarraj@ksu.edu.sa

\section{Amr Tolba}

Computer Science Department, Community College, King Saud University, Saudi Arabia, atolba@ksu.edu.sa

\section{Yongjun Ren*}

School of Computer and Software, Nanjing University of Information Science \& Technology, China,

renyj100@126.com

With the development of the Internet of Things (IoT), a large-scale, heterogeneous, and dynamic distributed network has been formed among IoT devices. There is an extreme need to establish a trust mechanism between devices, and blockchain can provide a zero-trust security framework for IoT. However, the efficiency of the blockchain is far from meeting the application requirements of the IoT, which has become the biggest resistance to the application of the blockchain in the IoT. Therefore, this paper combines sharding to build an effective Blockchain-based IoT data storage scheme (S-BDS). Sharding can solve the problem of blockchain capacity and scalability. While the blockchain provides data immutability and traceability for the IoT, it also brings huge demand for data credibility verification. The communication delay in the IoT system seriously affects the security of the system, while the Merkle proof of traditional blockchain occupies a lot of communication resources. This paper constructs Insertable Vector Commitment (IVC) in the bilinear group and replaces the Merkle tree with IVC to store IoT data in the blockchain. The construct has small-sized proof. It also has the ability to record the number of updates, which can prevent replay-attacks. Experiments show that each block processes 1000 transactions, the proof size of a single data piece is $30 \%$ of the original scheme, and proofs from different shards can be aggregated. IVC can effectively reduce communication congestion and improve the stability and security of the IoT system.

CCS CONCEPTS - Security and privacy Cryptography Public key (asymmetric) techniques Digital signatures -Information systems $\sim$ Data management systems $\sim$ Data structures $\bullet$ Mathematics of computing Discrete mathematics Additional Keywords and Phrases: Blockchain, Zero-trust, Internet of Things, Date Storage, Cryptographic Commitment

Permission to make digital or hard copies of all or part of this work for personal or classroom use is granted without fee provided that copies are not made or distributed for profit or commercial advantage and that copies bear this notice and the full citation on the first page. Copyrights for components of this work owned by others than ACM must be honored. Abstracting with credit is permitted. To copy otherwise, or republish, to post on servers or to redistribute to lists, requires prior specific permission and/or a fee. Request permissions from permissions@acm.org.

(C) 2022 Copyright held by the owner/author(s).

1533-5399/2022/1-ART1 $\$ 15.00$

http://dx.doi.org/10.1145/3511902 


\section{INTRODUCTION}

The IoT a significant part of the new generation of information technology. Today, the IoT has been widely used in various field, such as medical systems, power systems, transportation systems, and industrial systems [1-6]. With the wide application of the IoT, the complexity of the IoT network continues to increase. In this complex environment, the traditional peripheral-based security model is not applicable. Combining zero-trust and blockchain is considered an effective way to solve the appeal problem. Blockchain can ensure data security, and zero-trust adherence to the "never trust always verify" principle can improve access management and user authentication. Blockchain can also further enhance zero-trust security [7-11]. The combination of zero trust and blockchain has attracted the attention of many scholars. Sultana et al. built a medical image sharing system using blockchain and the zero-trust principle [7]. Zhao et al. proposed the use of blockchain to strengthen the security of identity authentication in the ZIoT (Zero Trust Internet of Things) [8]. Dhar et al. constructed a security framework for IoT devices based on zero trust and blockchain [10]. However, these applications directly use the traditional blockchain, and the problems of low throughput and high verification overhead of the blockchain have not been solved.

The two shortcomings of the blockchain's low throughput and high verification overhead have become obstacles to the application of blockchain in ZIoT. This article focuses on how to increase throughput and reduce communication consumption when verifying data correctness. First of all, the concept of blockchain sharding is introduced in the storage mechanism of this article. Simply put, sharding is to divide the blockchain nodes into different shards, so that the nodes of different shards maintain different sub-chains. In this way, each node only needs to process part of the transaction, and different shards can process transactions in parallel. The throughput of the blockchain can also be greatly improved [12,13]. Secondly, we changed the data structure of blockchain storage. In the traditional blockchain, data is stored through the Merkle tree. The correctness and completeness of the data are verified by Merkle proof. The Merkle proof is the hash sequence from the data piece to be verified to the Merkle root, and the Merkle root needs to be reconstructed through a series of hash operations during proof verification. But the proof size of the storage scheme based on Merkle tree is very large. Moreover, the Merkle tree cannot implement batch processing of storage proofs. When multiple pieces of data need to be certified, the proof can only be generated separately for each data piece $[14,15]$. The principle of "verify and never trust" should be upheld in IoT applications under the framework of zero trust. A large amount of data needs to be verified for correctness and completeness. When the amount of proof data is large, the linearly increasing proof size will bring great pressure to the transmission of the proof. Frequent data verification is required in the zero-trust architecture. The large-size proof may cause network delays and affect the normal operation of the IoT system $[16,17]$. In order to reduce the size of the proof and improve the communication efficiency of the system, this paper proposes to replace the Merkle tree with Insertable vector commitments.

\section{Our Contribute:}

The focus of this paper is to reduce the communication consumption and improve the throughput of the blockchain. It can be better applied in the context of ZIoT. The specific contributions are as follows:

- First, we proposed an effective blockchain-based data storage scheme. Unlike traditional blockchain systems, in the ZIoT, only verified (authorized) devices/users can access data.

- Second, in order to improve the throughput of the blockchain, we shard the blockchain. The throughput can be effectively improved after sharding

- Third, we propose to use IVC to replace Merkle tree in the blockchain data layer. Compared with the traditional scheme, the scheme in this paper can not only realize the proof aggregation within the same shard, but also realize the proof aggregation between different shards. 
- Fourthly, this paper constructs IVC in bilinear group. After the analysis and comparison of the schemes, this scheme has small fixed size proofs in the verification of single data, multiple data in the same shard and multiple data in different shards.

The rest of this paper is organized as follows. Section 2 introduces the knowledge about ZIoT, blockchain and cryptography commitment. Section 3 analyzes the challenges faced by the application of blockchain in the IoT. Section 4 will introduce our data storage scheme. Section 5 will compare and analyze this scheme with other schemes. Finally, we conclude in Section 6.

\section{RELATED WORK}

In this section, we will describe in detail the work that has been done so far. First, we introduced the main architecture of the ZIoT and blockchain. Then we introduce the basic concept and development of cryptography commitment.

\subsection{ZloT and Blockchain}

The IoT is the "Internet of Everything". The IoT has become the forefront of the development of information technology and has also been widely used. The most basic structure of the IoT has three logical layers, namely the perception layer, the network layer and the application layer [18-20]. The perception layer is to obtain the required information through the sensor network, and is the source of the IoT to collect information. The network layer is the link that connects the perception layer and the application layer. The data collected by the perception layer is transmitted to the application layer through the network layer, and the instructions issued by the application layer are also transmitted to the perception layer through the network layer. The application layer is the core part of the IoT system. The application layer obtains a large amount of data from the perception layer through the network layer, and analyzes and processes the obtained data according to application requirements [21-24]. Figure 1 is the architecture diagram of the IoT.

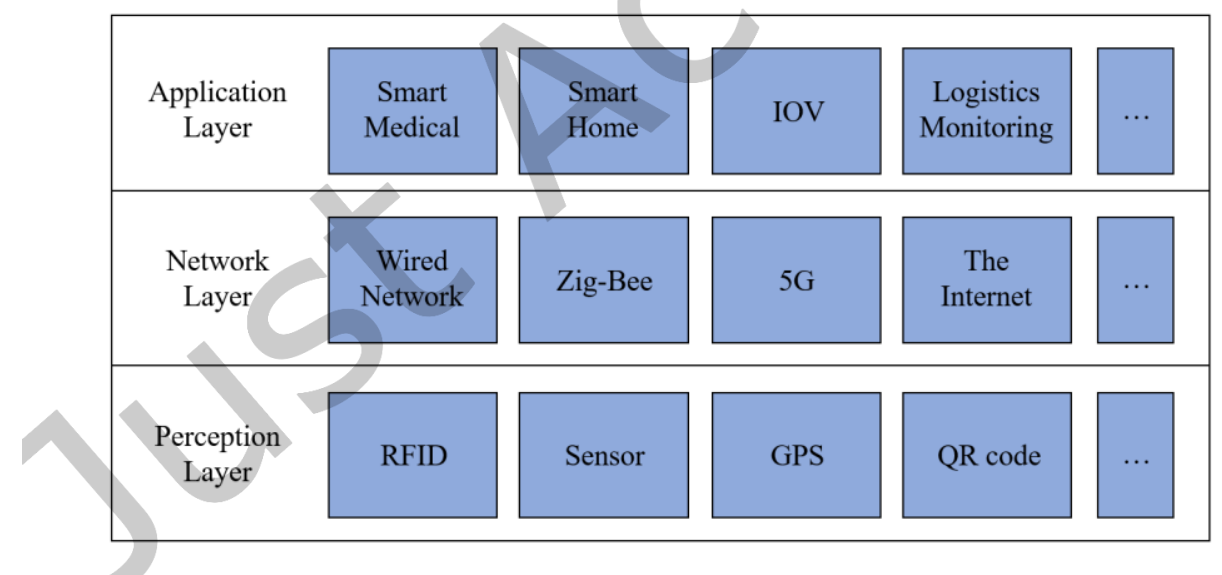

Fig. 1: Architecture of loT

The traditional peripheral-based security model divides the network into two parts: the internal network and the external network. It is usually assumed that the internal network is trust and the external network is not. This traditional security model is no longer suitable in the complex network environment of the IoT. The zero-trust security model is different from the traditional security model based on the peripheral. It cancellations the network 
peripheral. The zero-trust security model rigorously validates all users or devices accessing IoT data, both inside and outside the network. In a zero-trust security model, no user or device should be inherently trusted. All entities need to be authenticated, authorized, and monitored before accessing data $[7,8]$.

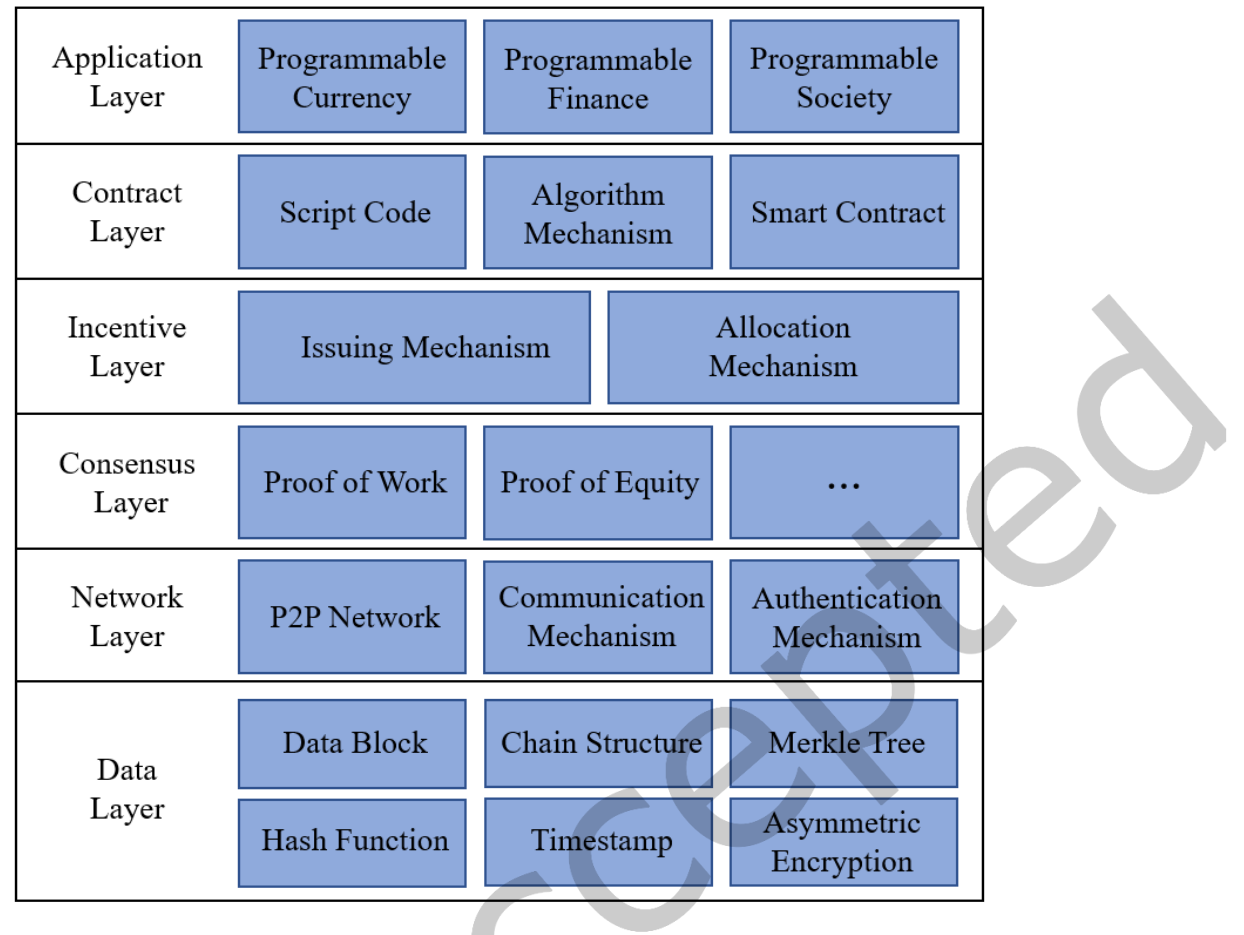

Fig. 2: Architecture of the blockchain

Blockchain is a special database technology, which uses distributed storage and combines point-to-point transmission, consensus algorithm, cryptography algorithm and other technologies [25-27]. The blockchain architecture is divided into six layers: the data layer, the network layer, the consensus layer, the incentive layer, the contract layer, and the application layer [28,29]. At the bottom of the architecture is the data layer. The implementation of accounts and various blockchain transactions requires the use of digital signatures, Hash functions and other cryptography techniques. The network layer is responsible for the connection and communication between nodes. The consensus layer encapsulates the consensus algorithm and the consensus mechanism, which enables the scattered nodes to reach a consensus on the validity of data. The incentive layer mainly includes the issuing mechanism and distribution mechanism of economic incentives. The contract layer encapsulates a variety of scripts, algorithms, and smart contracts. It gives blockchain programmable features. The application layer includes a variety of applications for blockchain. A blockchain application necessarily includes the data layer, the network layer, and the consensus layer. The incentive layer, contract layer and application layer are not necessary for every blockchain application. Figure 2 is the architecture of blockchain. Many scholars have used blockchain to protect data security in the IoT [30-33]. The principle of zero trust is "verification never trust", so the user or device needs to be verified before gaining access rights. In the ZIoT, the blockchain can perform individual identity verification on IoT devices or users without a central organization. Each entity can register its identity in the blockchain using key information such as 
user ID, device number, etc. It can then be verified against its previously registered hash on the blockchain. Only authenticated nodes can access data.

\subsection{Cryptography Commitment}

Commitment is one of the most basic cryptology protocols. A commitment can be seen as a sealed letter. If a party needs to commit to a message, it can put the information in the envelope, and when it needs to be made public, it just needs to open the envelope. In this process, two conditions need to be met, namely, Hiddenness and binding. Hiddenness refers that no one except the committer can access the message in the envelope, and binding refers that the message opened can only be the message that was originally placed in the envelope [34-35].

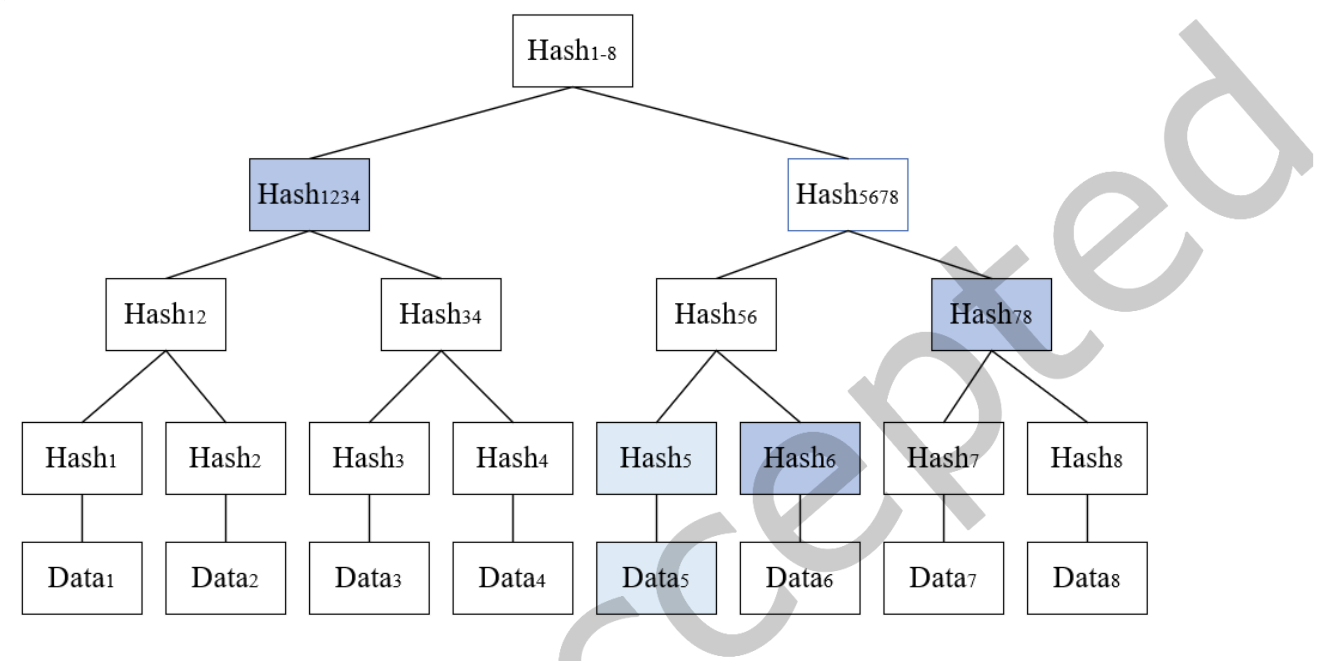

Fig. 3: Merkle tree

Vector commitment is a special commitment. It can commit a vector with a length of $\mathrm{n}$, and then open the commitment at any position. The concept of vector commitment first appeared in [14,36,37]. Catalano et al. instantiated vector commitment [38]. They use CDH (computational Diffie-Hellman) and RSA (Rivest-SharmirAdleman) assumptions to build schemes. The characteristic of Catalano's scheme is that the size of the commitment and the size of the proof are independent of the vector length n. Later, Lai et al. expanded Catalano's scheme, proposed and formalized the sub-vector commitment, and realized the I-sub-vector proof of constant proof size [39]. Boneh et al. built vector commitments on hidden-order groups, and their scheme was the first to allow multiple proofs to be aggregated under certain conditions, and it is the first scheme with constant-size public parameter [40]. Alin et al. used polynomial commitments and Lagrange polynomials to build a vector commitment scheme. They use it in accountbased stateless cryptocurrencies [41]. Gorbunov et al. extended the scheme [39] to achieve the aggregation of proof between different commitments and applied it to smart contracts [42]. Key-value commitment is proposed by Shashank et al. Key-value commitment is similar to vector commitment. A key-value commitment allows one to commit to a key-value map. Then enter the specific key to open the commitment. But their scheme cannot aggregate proofs indefinitely [43].

Merkle tree can also be considered a vector commitment, and the root of the Merkle tree is a commitment value [14]. The message committed by the Merkle tree is all its leaf nodes. All sibling nodes in the path from the leaf node to the root node and leaf nodes themselves form an opening proof of vector commitment. Traditional blockchain uses the 
Merkle tree's opening proof to verify the integrity and correctness of the data stored in the blockchain. Figure 3 is an example of a Merkle tree. In Figure 3, the light blue squares represent the data to be verified, and the set of dark blue squares is the corresponding Merkle proof.

\section{THE SHORTCOMINGS OF TRADITIONAL BLOCKCHAIN}

Blockchain technology can provide a secure data storage and network environment for the IoT. But the storage capacity and throughput of blockchain is a huge barrier to its organic integration with IoT systems. Traditional blockchains are mostly single-chain structures, with all miners competing with each other for the right to generate the latest block. For example, Bitcon generates on average one block every 10 minutes. In order to ensure the security of the system, the system generates blocks at a fixed time. As more miners join the scheme, the difficulty of mining will increase. Moreover, as the number of joining nodes increases, so does the time to reach a consensus on the system [4446]. In addition, full nodes need to store the data of the entire network. Tens of thousands of bytes of data can be collected in real time in the perceptual layer of the IoT, but the throughput and storage capacity of the blockchain cannot meet the needs of the IoT [47-49].

Sharding technology is a powerful tool to address the storage capacity and scalability of blockchain. The main idea of sharding technology is to divide the blockchain network into subnetworks. Each subnetwork contains a subset of nodes, which is a "shard". After sharding, the system runs multiple blockchains in parallel, and the beacon chain is responsible for maintaining all operations of the whole system [50-52]. At the same time, the transactions that the blockchain system needs to process are also divided according to the sharding rules. This allows each node to process only a subset of transactions, and different shards can process transactions in parallel. This lets throughput to increase linearly with the number of shards. In addition, the storage node only needs to store the transactions of its own shard, which greatly reduces the storage pressure of the storage node.

Traditional blockchain systems use Merkle trees to store data, but the proof of the correctness and completeness of the data is very large. The sharded data is also stored in different shards, but the storage mechanism based on the Merkle tree cannot aggregate the proofs. When verifying data from different shards, we can only generate separate proofs for each data. A huge amount of data verification demands a huge amount of Merkle proofs. This will generate huge communication pressure, resulting in end-to-end communication delay, and thus jeopardize the security of the IoT system.

\section{S-BDS IN ZERO-TRUST IOT}

This paper constructs an S-BDS in ZIoT. As shown in Figure 4, unlike traditional blockchain systems, the ZIoT environment needs to follow the "verify and never trust" principle. Authentication is required when users need to access data of storage nodes or when data aggregator nodes transfer data into the storage nodes. The data collected by the sensor node is transmitted to the distributed data storage center by the aggregator node. The data in the storage center can be used to implement various IoT applications. The data storage node acts as a full node in the block system, and the aggregator node acts as a light node in the blockchain system. The node maintains the blockchain through a consensus protocol. In order to improve the efficiency of the system, we split the blockchain network. We divide the entire blockchain network into several sub-networks, and the transaction processing in the blockchain network will also be divided into different shards for processing, so that each node only needs to process a part of the transaction, and different nodes can perform Parallel processing can increase the speed of transaction processing and verification and increase the throughput of the network. The authentication chain is responsible for storing user 
information that can access the system. In addition, in order to ensure the security of the blockchain system, the node also needs to maintain a Beacon chain. Beacon chain is responsible for the operations required to maintain the entire network, such as generating random numbers, processing POS (proof of stake), rebalancing shards, etc.

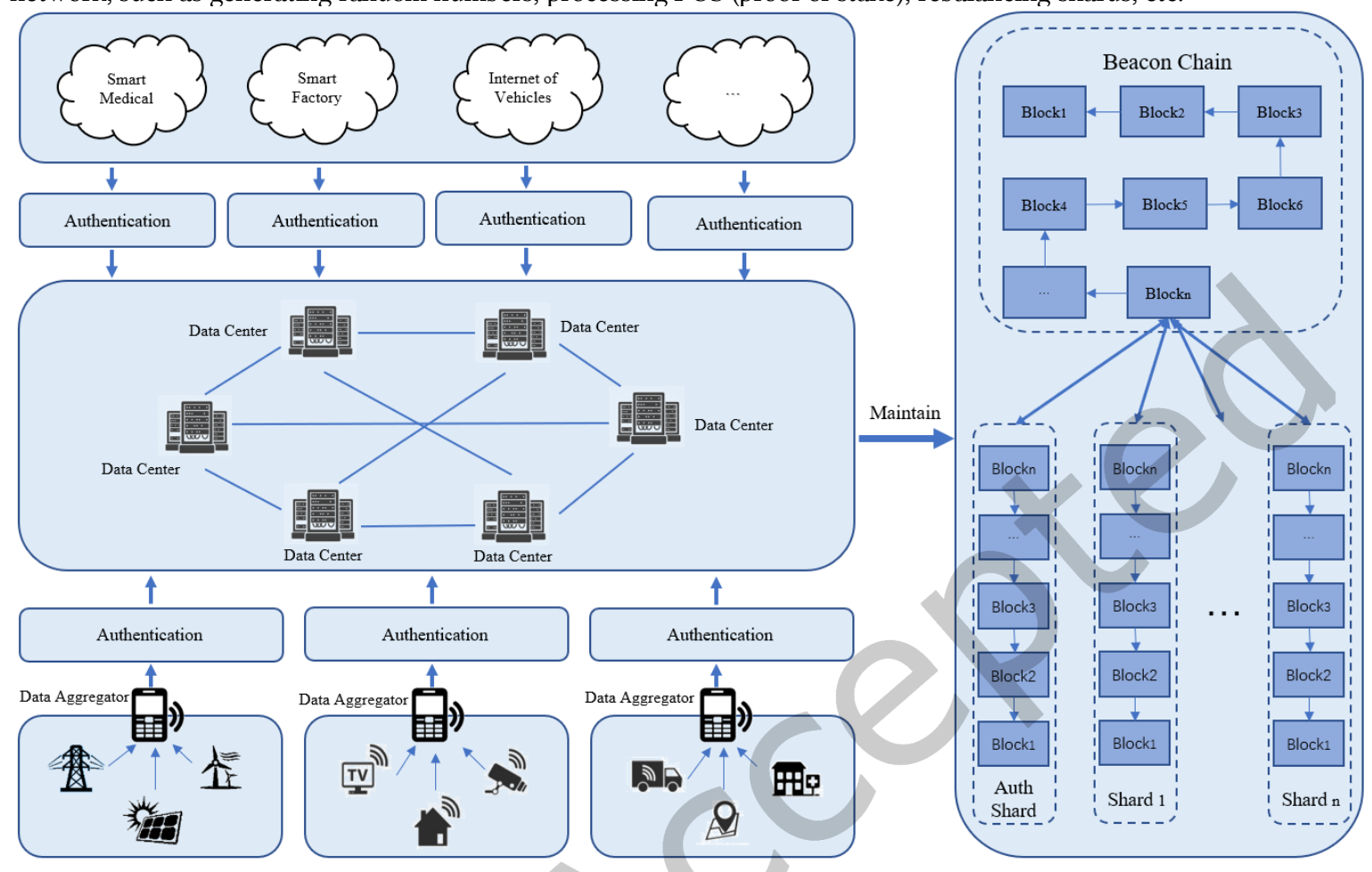

Fig. 4: Framework of S-BDS

The zero-trust device authentication process in this paper is a modification of Zhao et.al. scheme [8]. As shown in Figure 5, users and devices need to register with the authentication chain with their key information, such as ID or device serial number. The authentication chain is responsible for storing user information that can access the system. The registered user and device are assigned a public key, which is recorded in the authentication chain. To access a storage node, perform the following steps:

1) In this scheme, the blockchain is processed by shards, so the storage node only stores the data of its own shards. Before accessing data, user/device needs to retrieve the public key of the storage node from the authentication chain. It then encrypts its address in the blockchain with the public key of the storage node and sends it to the storage node.

2) After receiving the request, the storage node uses its own private key to decrypt it. The public key of user/device is then searched in the blockchain, and if public key exists on the blockchain, the randomly generated string and a timestamp are encrypted using the device's public key. Finally, the encrypted information is returned to the user/device.

3) The user/device uses its private key to decrypt the ciphertext to obtain the string and time stamp, and then signs the string and time stamp with the private key and sends them back to the storage node. 
4) The storage node decrypts the string using the user's public key and verifies whether the string is randomly generated in Step 2. After the authentication succeeds, the authenticity of the access is confirmed.

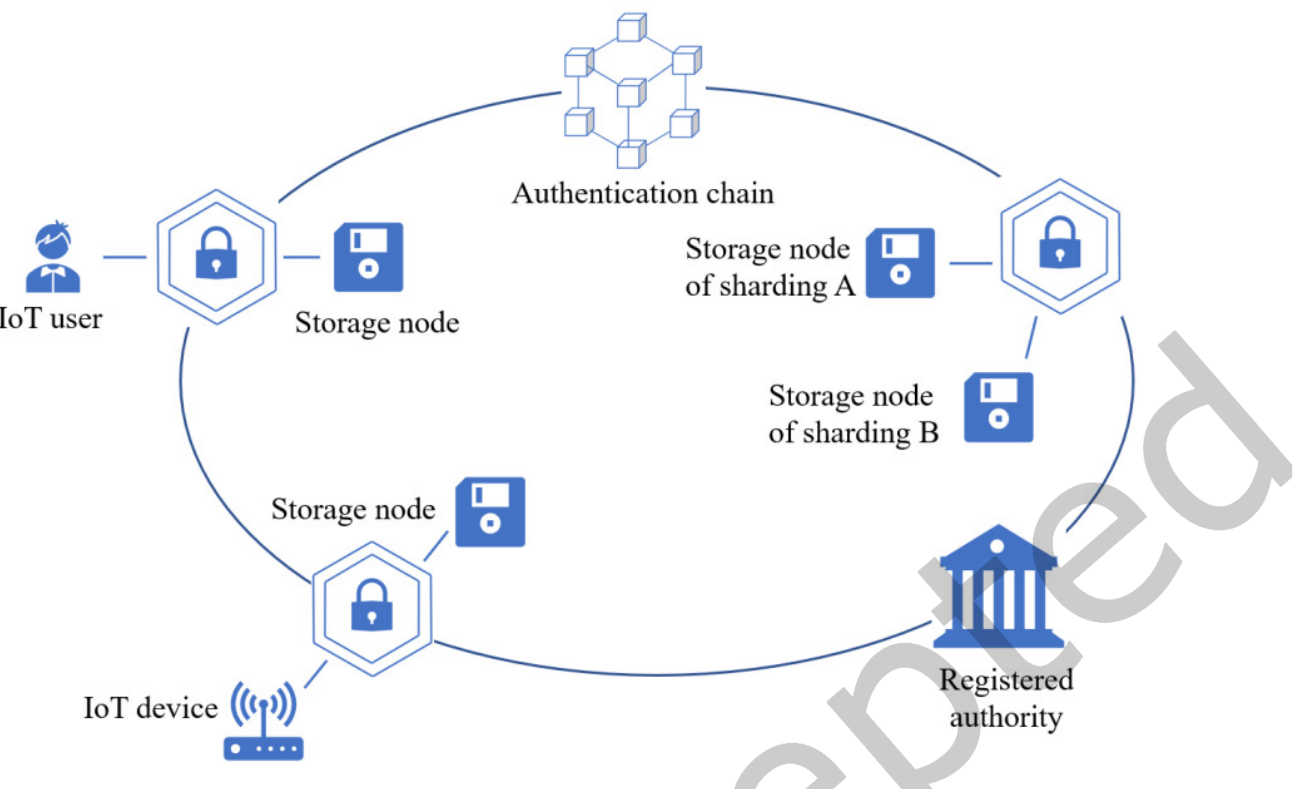

Fig. 5: Blockchain enabled zero trust loT user/device authentication

\subsection{IVC-based Storage Mechanism}

The IVC is used to reduce communication costs when verifying data correctness. In the blockchain data storage mechanism based on the Merkle tree, when verifying the integrity and correctness of the data, it is necessary to provide the Hash sequence from the data piece to be verified to the Merkle root as the storage proof. The blockchain storage mechanism based on the Merkle tree cannot realize batch processing of proofs. When dealing with multiple pieces of data, it can only generate multiple proofs separately. The storage proof size is related to the number of data pieces to be validated and the depth of the Merkle tree. In order to reduce the proof size and improve the communication efficiency, this paper proposes to use IVC to replace the Merkle tree. The scheme in this paper not only reduces the size of a single proof, but also aggregates proofs of different shards into a single proof, through which the correctness and integrity of the data on different shards can be verified in batches.

Similar to the traditional blockchain, the block of the scheme in this paper is composed of two parts, namely the block head and block body. The full node is responsible for storing all the information of the block header and block body. Light nodes only need to store the information of the block header. The difference is that we replaced the Merkle tree structure in the ordinary blockchain with IVC. Figure 6 illustrates this structure: 

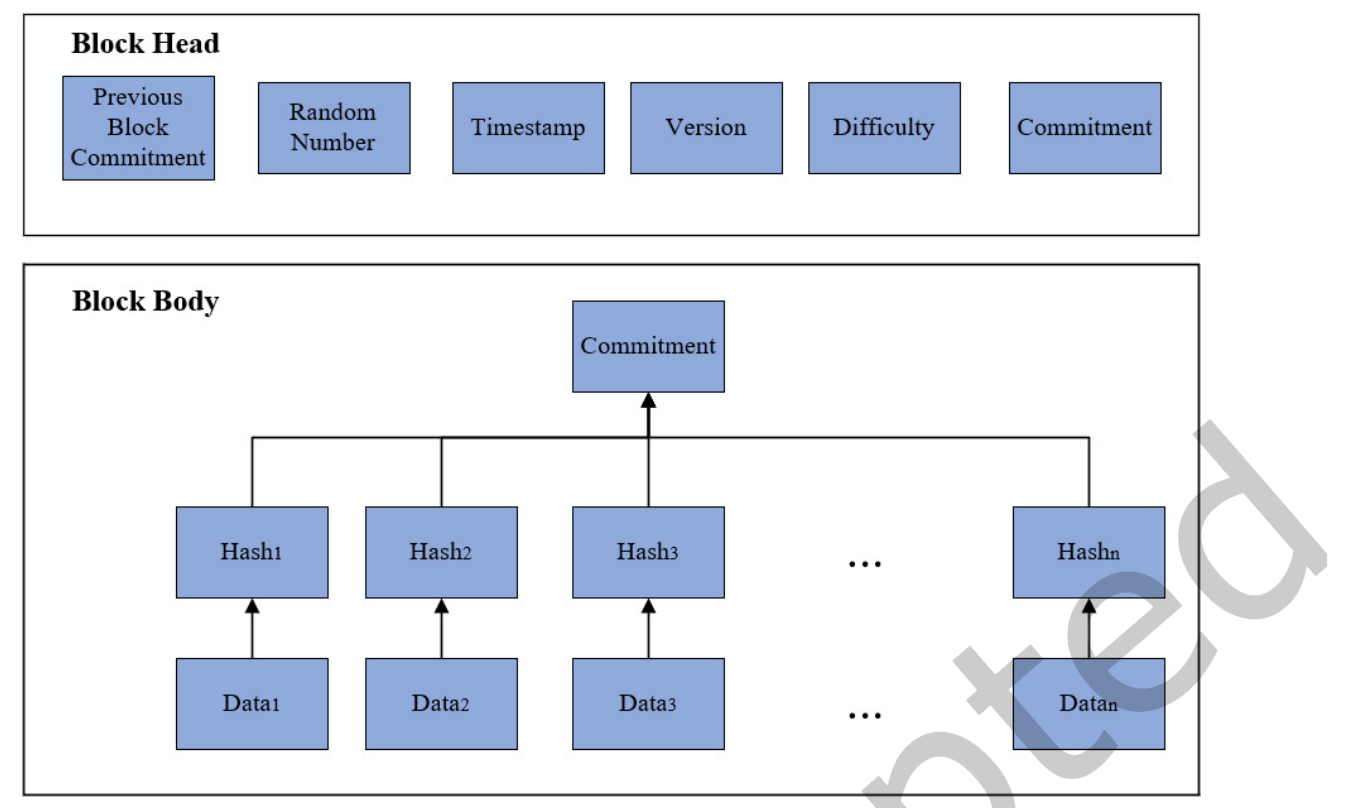

Fig. 6 : Blockchain data storage mechanism based on IVC

Some specific operations in the proposed mechanism are described as follows:

User registration: Nodes that want to access ZIoT need to register with the authentication chain through the registered authority. The registered authority assigns a pair of public and private keys to the newly registered node. Nodes with public and private key pairs are eligible to upload or access data, but each access must be verified. Nodes are divided into different shards by beacon chain. Nodes in the same shard only need to process transactions generated by their own shard. When the node uploads data, it needs to use the private key $S K$ to sign the transaction, and other nodes use the public key to verify the transaction and determine whether the transaction is sent by the owner of the private key.

Data storage transactions: When a node uploads data to a blockchain, the node needs to publish the Data upload transaction $T x=\left[D A T A, P K, u_{i}\right]$ (where DAT $A$ is the data to be stored, $P K$ is the user's public key, and $u_{i}$ is the storage times corresponding to $P K$.). The data can be encrypted to maintain its hidden properties. The node uses the private key $S K$ corresponding to the public key $P K$ to sign and publish the transaction, and then broadcasts to other nodes in the shard where the node is located.

Block generation: Blocks are generated by competition between storage nodes through consensus protocol. The storage node collects transactions broadcast on the blockchain network. After the storage node collects enough legal data, all the data is generated into a key-value pair set $M$, and the Insert algorithm is called to generate a commitment. The storage node puts the calculated commitment value into the block header, and packs all collected transactions into the block body.

proof generation: When you need to verify the integrity and correctness of the data piece. Storage nodes can convince users that the data to be validated is indeed stored in the blockchain by generating proof of data storage. In order to verify the proof, the user needs to store the corresponding block header information, the 
storage node needs to return the corresponding data, and provide the data storage proof generated by the open algorithm.

Proof aggregation: When the correctness and completeness of data need to be verified, the data may be stored in different shards. By calling the aggregation algorithm (SCA. Agg or MCA.Agg), we can aggregate the proofs of data from different shards. Using a single proof can verify the correctness and completeness of multiple data in batches. The aggregation function can effectively reduce the communication consumption of the proof. Figure 7 shows an example of proof aggregation.

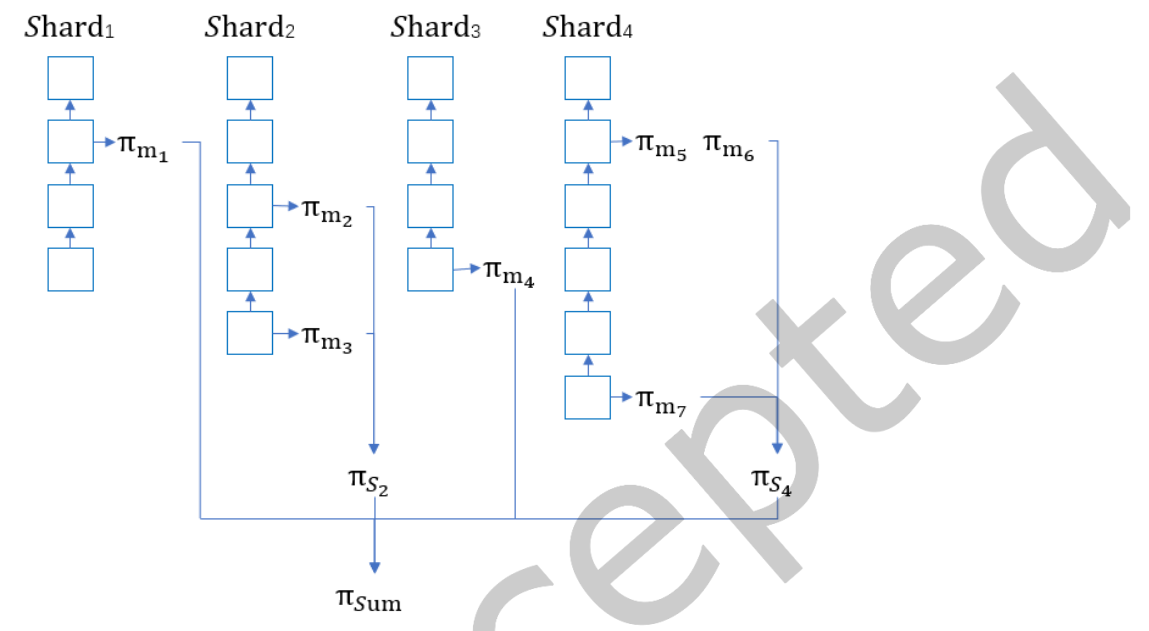

Fig. 7: Proof of aggregation

\subsection{Insertable Vector Commitment}

\subsubsection{Preliminaries}

Notation: This paper, uses $\lambda \in \mathbb{N}$ to denote security parameters, let negl( $\cdot)$ denote any negligible function, and uses $\operatorname{poly}(\cdot)$ to denote any polynomial whose upper bound is a certain univariate polynomial. If algorithm $A$ is modeled as a probabilistic Turing machine running at time $\operatorname{ploy}(\lambda)$, it is called a polynomial time algorithm.

Bilinear Pairing: Let $G_{1}, G_{2}$ be an additive cyclic group of order $p, G_{T}$ is a multiplicative cyclic group of the same order. Then, define a bilinear mapping $e: G_{1} \times G_{2} \rightarrow G_{T}$. Where $p$ is a prime number. Integer $a, b \in Z_{p}^{*}$, then the bilinear mapping satisfies the following three properties:

- Bilinearity: $\exists A \in G_{1}, B \in G_{2}$ and $a, b \in Z_{p}^{*}, \exists e\left(A^{a}, B^{b}\right)=e(A, B)^{a b}$.

- Non-degeneracy: $\exists A \in G_{1}, B \in G_{2}$, such that $e(A, B) \neq 1 ; g_{1}$ and $g_{2}$ are generators of $G_{1}$ and $G_{2}$, respectively. $e\left(g_{1}, g_{2}\right)$ is the generator of $G_{T}$.

- Computability: For any $A \in G_{1}, B \in G_{2}$, there are effective algorithms so that the result of $e(A, B)$ can be obtained in polynomial time.

When $G_{1}=G_{2}$, the bilinear mapping is said to be symmetric.

l-wBDHE (Weak bilinear Diffie-Hellman exponent) Assumption: Let $\left(P, G, G_{T}, e, g\right)$ be a uniformly randomly generated bilinear pair parameters. Given $\left(g_{1}^{\alpha}, g_{1}^{\alpha^{N}}, g_{1}^{\alpha^{N+2}}, \ldots, g_{1}^{\alpha^{2 N}} ; g_{2}^{\alpha}, \ldots, g_{2}^{\alpha^{N}} ; g_{T}^{\alpha^{N+1}}\right)$, calculating $g_{1}^{\alpha^{N+1}}$ is difficult. 


\subsubsection{System model}

This section details the model of IVC. In order to better meet the above application requirements, the scheme in this paper adds Insert algorithm compared with the traditional vector commitment. The concept of key-value pair in keyvalue commitment is introduced into the commitment scheme of this paper. The user's public key is mapped by key, and the data piece is represented by value. The scheme in this paper does not need to set the commitment number of each block in advance before building blocks. An insertable vector commitment scheme consists of the following seven polynomial time algorithms.

$\operatorname{Setup}\left(\mathbf{1}^{\lambda}\right)$ : Given the security parameter $\lambda$, the algorithm outputs the public parameters $P P$ and the initial commitment $C$.

Insert $(\boldsymbol{C}, \boldsymbol{M}, \boldsymbol{U},(\boldsymbol{k}, \boldsymbol{m}))$ : This algorithm is used to insert new elements $(k, m)$ into the commitment set $M, U$. Input commitment $C$, the set $M$ of the key-value pair, the set $U$ of insertion times and key-value pair $(k, m)$.This algorithm outputs the updated commitment $C^{\prime}$, updated set $M^{\prime}$ and $U^{\prime}$. $(k, m)$ corresponding proof $\pi_{k}$ and an updated information aux.

$\operatorname{Open}\left(\boldsymbol{C}, \boldsymbol{M}, \boldsymbol{U}, \boldsymbol{k}_{\boldsymbol{i}}\right)$ : This algorithm is used to generate proofs corresponding to $k_{i}$. Input commitment $C$, the set $M$ of the key value pair, the set $U$ of insertion times, and the key $k_{i}$. This algorithm outputs $k_{i}$ corresponding to the proof $\pi_{i}$.

$\boldsymbol{S C A} \boldsymbol{A} \boldsymbol{g} \boldsymbol{g}\left(\boldsymbol{C}, \boldsymbol{\pi}_{\boldsymbol{i}, \boldsymbol{i} \in \boldsymbol{S}}, \boldsymbol{S}, \boldsymbol{V}\right)$ : This algorithm is used to aggregate the proofs corresponding to the subset $S$. Input commitment $C$, the proof $\pi_{i}$ to aggregate, the set $S$ of key-value pairs to be proved and the set $V$ of insertion times. This algorithm outputs the corresponding proof $\pi_{S}$.

$\boldsymbol{S C A} \operatorname{Ver}\left(\pi_{\boldsymbol{S}}, \boldsymbol{S}, \boldsymbol{V}, \boldsymbol{C}\right)$ : This algorithm is used to verify that $\pi_{\mathrm{S}}$ is valid. Input commitment $C$, the set $S$ of key-value pairs to be proved, the set $V$ of insertion times. and the corresponding aggregate proof $\pi_{S}$. This algorithm outputs 0 or 1 . The validation succeeds when 1 is printed, and fails when 0 is printed.

$\boldsymbol{M C A} \cdot \boldsymbol{A g} \boldsymbol{g}\left(\left(\boldsymbol{C}_{\boldsymbol{j}}, \boldsymbol{\pi}_{j, i}, \boldsymbol{S}_{\boldsymbol{j}}, \boldsymbol{V}_{\boldsymbol{j}}\right)_{\boldsymbol{j} \in \boldsymbol{L}}\right)$ : This algorithm is used to aggregate proofs from different commitments, and the number of aggregated commitments is $L$. Input commitment $C_{j}$, the proof $\pi_{j, i}$ to be aggregated, the set $S_{j}$ of key-value pairs to be proved and the set $V_{j}$ of insertion times. This algorithm outputs the corresponding proof $\pi$.

$\boldsymbol{M C A} \operatorname{Ver}\left(\left(\boldsymbol{C}_{\boldsymbol{j}}, \boldsymbol{S}_{\boldsymbol{j}}, \boldsymbol{V}_{\boldsymbol{j}}\right)_{\boldsymbol{j} \in \boldsymbol{L}}, \boldsymbol{\pi}\right)$ : This algorithm is used to verify that $\pi$ is valid. Input commitment $C_{j}$, the set $S_{j}$ of key-value pairs to be proved, the set $V_{j}$ of insertion times. and the corresponding aggregate proof $\pi$. The algorithm outputs 0 or 1 . This validation succeeds when 1 is printed, and fails when 0 is printed.

Definition 1 (SCA-binding): Given a security parameter $\lambda \in \mathbb{N}$, If for any PPT adversary $A$, satisfying:

$\operatorname{pr}\left[\begin{array}{c}\operatorname{Ver}\left(p p, C, S_{b}, V_{b}, \pi_{b}\right)=1 \\ \text { for both } b=0 \text { and } b=1 \\ \wedge x \neq x^{\prime} ; x, x^{\prime} \in S_{0} \cap S_{1} \\ \wedge y \neq y^{\prime} ; y, y^{\prime} \in V_{0} \cap V_{1}\end{array}|| \begin{array}{c}\operatorname{Setup}\left(1^{\lambda}, M\right) \rightarrow p p \\ A(p p) \rightarrow\left(C, S_{b}, V_{b}, \pi_{b}\right) \\ \text { for both } b=0 \text { and } b=1\end{array}\right] \in \operatorname{negl}(\lambda)$.

Then the scheme has binding.

Definition 2 (MCA-binding): Given a security parameter $\lambda \in \mathbb{N}$, If for any PPT adversary $A$, satisfying: 
$\operatorname{pr}\left[\begin{array}{c|c}\operatorname{Ver}\left(p p,\left(C_{j, b}, S_{j, b}, V_{j, b}\right)_{j \in L^{\prime}}, \pi_{b}\right)=1 \\ \quad \text { for both } b=0 \text { and } b=1 \\ \wedge x \neq x^{\prime} ; x, x^{\prime} \in S_{j, 0} \cap S_{j, 1} \\ \wedge y \neq y^{\prime} ; y, y^{\prime} \in V_{j, 0} \cap V_{j, 1}\end{array} \mid \begin{array}{c}\operatorname{Setup}\left(1^{\lambda}, M\right) \rightarrow p p \\ A(p p) \rightarrow\left(\left(C_{j, b}, S_{j, b}, V_{j, b}\right)_{j \in L}, \pi_{b}\right) \\ \text { for both } b=0 \text { and } b=1\end{array}\right] \in \operatorname{negl}(\lambda)$.

Then the scheme has binding.

Definition 3 (Correctness): Given a security parameter $\lambda \in \mathbb{N}$, For any safety parameter $\lambda$, any key-value pair set $M$, insertion times set $U$, and any subset $S \in M$ and $V \in U$.satisfying:

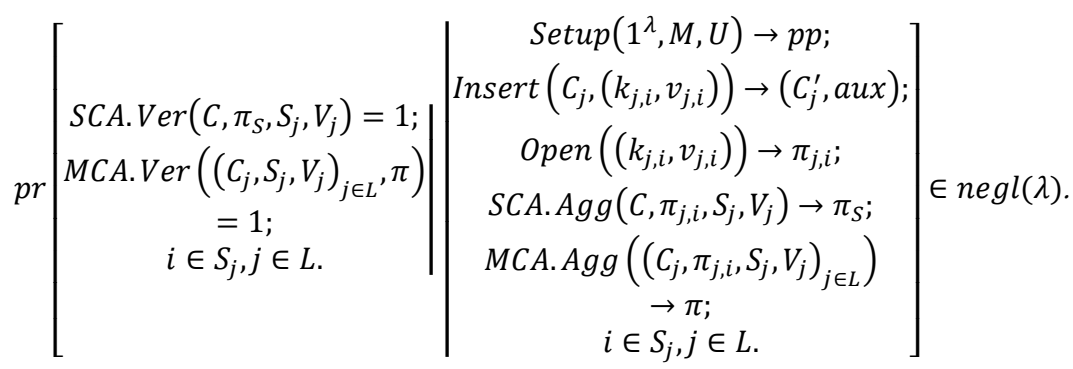

Then the scheme has correctness.

\subsubsection{IVC Based on Bilinear Mapping}

This section describes in detail the specific construction of IVC based on bilinear mapping. This scheme Starting from the [42], adds Insert algorithm and the function of recording the insert times of each key $k_{i}$ :

$\operatorname{Setup}\left(\mathbf{1}^{\lambda}\right)$ : Given a security parameter $\lambda \in \mathbb{N}$, This algorithm randomly generates bilinear pairs $e: G_{1} \times G_{2} \rightarrow G_{T}$ and Corresponding generator $g_{1} \in G_{1}, g_{2} \in G_{2}, g_{T}=e\left(g_{1}, g_{2}\right) \in G_{\mathrm{T}}$. Randomly select the secret number $\alpha \in Z_{P}$, generate $p p:=\left(g_{1}^{\alpha}, g_{1}^{\alpha^{N}}, g_{1}^{\alpha^{N+2}}, \ldots, g_{1}^{\alpha^{2 N}} ; g_{2}^{\alpha}, \ldots, g_{2}^{\alpha^{N}} ; g_{T}^{\alpha^{N+1}}\right)$. Choose two hash functions $H_{1}, H_{2}$. Set the public parameter $P P:=$ $\left\{p p, H_{1}, H_{2}\right\}$. When registering a new user, this algorithm needs to map $k_{i}$ to the unused $g_{1}^{\alpha^{i}}$. Generate set $M=\left\{\left(k_{i}, m_{i}\right)\right\}_{i \in[n]}$, set $U=\left\{\left(k_{i}, u_{i}\right)\right\}_{i \in[n]}$.

Insert $(\boldsymbol{C}, \boldsymbol{M}, \boldsymbol{U},(\boldsymbol{k}, \boldsymbol{m}), \boldsymbol{p p})$ : This algorithm outputs the updated commitment $C^{\prime},(k, m)$ corresponding proof $\pi_{k}$ and an updated information aux.

1) Update set $M^{\prime}=M \cup\left(k_{i}, m_{i}\right) \cdot u_{i}^{\prime}=u_{i}+1$.

2) Parsing commitment $C:=\left(C_{1}, C_{2}\right)=\left(g_{1}^{\sum_{k_{i} \in M} m_{i} \alpha^{i}}, g_{1}^{\sum_{k_{i} \in U} u_{i} \alpha^{i}}\right)$, calculation

$$
C^{\prime}:=\left(C_{1}^{\prime}, C_{2}^{\prime}\right)=\left(C_{1} \cdot\left(g_{1}^{\alpha^{i}}\right)^{m_{i}}, C_{2} \cdot g_{1}^{\alpha^{i}}\right)
$$

Output the updated commitment $C^{\prime}:=\left(C_{1}^{\prime}, C_{2}^{\prime}\right)$.

3) Generate update information aux aux := $\left(k_{i}, u_{i}^{\prime}\right)$.

$\operatorname{Open}\left(\boldsymbol{C}, \boldsymbol{M}, \boldsymbol{U}, \boldsymbol{k}_{\boldsymbol{i}}, \boldsymbol{p p}\right)$ : This algorithm outputs $k_{i}$ corresponding to the proof $\pi_{i}$. calculation

$$
\pi_{i}:=\left(\pi_{i, 1}, \pi_{i, 2}\right)=\left(g_{1}^{\sum_{k_{j} \in M, k_{j} \neq k_{i}} m_{j} \alpha^{N+1-i+j}}, g_{1}^{\sum_{k_{j} \in U, k_{j} \neq k_{i}} u_{j} \alpha^{N+1-i+j}}\right)
$$

Output the proof $\pi_{i}:=\left(\pi_{i, 1}, \pi_{i, 2}\right)$. 
$\boldsymbol{S C A} \operatorname{Ag} \boldsymbol{g}\left(\boldsymbol{C}, \boldsymbol{\pi}_{\boldsymbol{i}, \boldsymbol{i} \in \boldsymbol{S}}, \boldsymbol{S}, \boldsymbol{V}, \boldsymbol{P P}\right)$ : This algorithm can aggregate proofs of multiple locations of a single commitment.

1) Calculation $a_{i, 1}=H_{1}\left(k_{i}, C_{1}, S\right), a_{i, 2}=H_{1}\left(k_{i}, C_{2}, V\right), b_{1}=H_{2}\left(C_{1}, S\right), b_{2}=H_{2}\left(C_{2}, V\right)$.

2) Output the proof

$$
\pi_{S}=\left(\prod_{k_{i} \in S} \pi_{i, 1}^{a_{i, 1}}\right)^{b_{1}} \cdot\left(\prod_{k_{i} \in V} \pi_{i, 2}^{a_{i, 2}}\right)^{b_{2}}
$$

The pseudo-code of the algorithm is as follows:

\section{ALGORITHM 1: SCA.Agg}

InPut: $C, \pi_{i, i \in S}, S, V, H_{1}, H_{2}, p p$

OutPut: $\pi_{S}$

for $k_{i} \in S$ do

if $k_{i} \in V$ then

compute $a_{i, 1}=H_{1}\left(k_{i}, C_{1}, S\right)$;

compute $a_{i, 2}=H_{1}\left(k_{i}, C_{2}, V\right)$;

else

return error and termination of the algorithm;

end

compute $b_{1}=H_{2}\left(C_{1}, S\right)$;

compute $b_{2}=H_{2}\left(C_{2}, V\right)$;

compute $\pi_{S}=\left(\prod_{k_{i} \in S} \pi_{i, 1}^{a_{i, 1}}\right)^{b_{1}}\left(\prod_{k_{i} \in V} \pi_{i, 2}^{a_{i, 2}}\right)^{b_{2}}$;

$\operatorname{Return} \pi_{S}$;

$\boldsymbol{S C A . V e r}\left(\boldsymbol{\pi}_{\boldsymbol{S}}, \boldsymbol{S}, \boldsymbol{V}, \boldsymbol{C}, \boldsymbol{P P}\right)$ : Verify the equation

$e\left(C_{1}, g_{2}^{\sum_{k_{i} \in S} \alpha^{N+1-i} a_{i, 1}}\right)^{b_{1}} e\left(C_{2}, g_{2}^{\sum_{k_{i} \in V} \alpha^{N+1-i} a_{i, 2}}\right)^{b_{2}}=e\left(\pi_{S}, g_{2}\right) g_{T}^{\alpha^{N+1}\left(\sum_{k_{i} \in S} m_{i} \cdot a_{i, 1} \cdot b_{1}+\sum_{k_{i} \in V} u_{i} \cdot a_{i, 2} \cdot b_{2}\right)}$

This algorithm outputs 0 or 1 . The validation succeeds when output $=1$, and fails when output $=0$.

The pseudo-code of the algorithm is as follows:

\section{ALGORITHM 2: SCA.Ver}

InPut: $\pi_{S}, S, V, C, H_{1}, H_{2}, p p$

OutPut: 0 or 1

$$
\begin{aligned}
& \text { for } k_{i} \in S \text { do } \\
& \text { if } k_{i} \in V \text { then }
\end{aligned}
$$

compute $a_{i, 1}=H_{1}\left(k_{i}, C_{1}, S\right)$;

compute $a_{i, 2}=H_{1}\left(k_{i}, C_{2}, V\right)$;

else

return error and termination of the algorithm;

end 


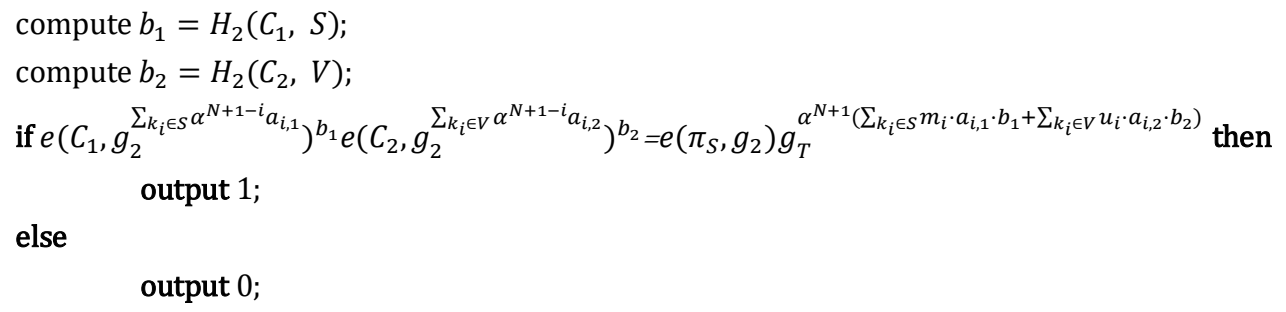

$\boldsymbol{M C A} \boldsymbol{A g} \boldsymbol{g}\left(\left(\boldsymbol{C}_{\boldsymbol{j}}, \boldsymbol{\pi}_{\boldsymbol{j}, \boldsymbol{i}}, \boldsymbol{S}_{\boldsymbol{j}}, \boldsymbol{V}_{\boldsymbol{j}}\right)_{\boldsymbol{j} \in \boldsymbol{L}}, \boldsymbol{P P}\right)$ : The algorithm can aggregate the proofs of different commitment

1) Calculation $a_{j, i, 1}=H_{1}\left(k_{j, i}, C_{j, 1}, S_{j}\right), a_{j, i, 2}=H_{1}\left(k_{j, i}, C_{j, 2}, V_{j}\right), b_{j, 1}=H_{2}\left(j, C_{j, 1}, S_{j}\right), b_{j, 2}=H_{2}\left(j, C_{j, 2}, V_{j}\right)$.

2) Output the proof

$$
\pi=\prod_{j \in L}\left(\left(\prod_{k_{j, i} \in S_{j}} \pi_{i, 1}^{a_{j, i, 1}}\right)^{b_{j, 1}} \cdot\left(\prod_{k_{j, i} \in V_{j}} \pi_{i, 2}^{a_{j, i, 2}}\right)^{b_{j, 2}}\right)
$$

The pseudo-code of the algorithm is as follows:

\section{ALGORITHM 3: MCA. Agg}

InPut: $\left(C_{j}, \pi_{j, i}, S_{j}, V_{j}\right)_{j \in L}, H_{1}, H_{2}, p p$

OutPut: $\pi$

for $j \in L$ do

for $k_{i} \in S$ do

if $k_{i} \in V$ then

compute $a_{j, i, 1}=H_{1}\left(k_{j, i}, C_{j, 1}, S_{j}\right)$;

compute $a_{j, i, 2}=H_{1}\left(k_{j, i}, C_{j, 2}, V_{j}\right)$;

else

end

return error and termination of the algorithm;

end

compute $b_{j, 1}=H_{2}\left(j, C_{j, 1}, S_{j}\right)$;

compute $b_{j, 2}=H_{2}\left(j, C_{j, 2}, V_{j}\right)$;

compute $\pi=\prod_{j \in L}\left(\left(\prod_{k_{j, i} \in S_{j}} \pi_{i, 1}^{a_{j, i, 1}}\right)^{b_{j, 1}} \cdot\left(\prod_{k_{j, i} \in V_{j}} \pi_{i, 2}^{a_{j, i, 2}}\right)^{b_{j, 2}}\right)$;

Return $\pi$;

$\operatorname{MCA.Ver}\left(\left(\boldsymbol{C}_{j}, \boldsymbol{S}_{j}, \boldsymbol{V}_{j}\right)_{j \in L}, \boldsymbol{\pi}, \boldsymbol{P P}\right):$ Verify the equation

$\prod_{j \in L} e\left(C_{j, 1}, g_{2}^{\sum_{k_{i} \in S_{j}} \alpha^{N+1-i} a_{j, i, 1}}\right)^{b_{j, 1}} e\left(C_{j, 2}, g_{2}^{\sum_{k_{i} \in V_{j}} \alpha^{N+1-i} a_{j, i, 2}}\right)^{b_{j, 2}=e}\left(\pi, g_{2}\right) g_{T}^{\alpha^{N+1} \sum_{j \in[L]}\left(\sum_{k_{i} \in S_{j}} m_{j, i} \cdot a_{j, i, 1} \cdot b_{1}+\sum_{k_{i} \in V_{j}} u_{j, i} \cdot a_{j, i, 2} \cdot b_{2}\right)}$.

This algorithm outputs 0 or 1 . The validation succeeds when output $=1$, and fails when output $=0$.

The pseudo-code of the algorithm is as follows: 


\section{ALGORITHM 4: MCA.Ver}

InPut: $\left(\boldsymbol{C}_{\boldsymbol{j}}, \boldsymbol{S}_{\boldsymbol{j}}, \boldsymbol{V}_{\boldsymbol{j}}\right)_{\boldsymbol{j} \in \boldsymbol{L}}, \boldsymbol{\pi}, H_{1}, H_{2}, p p$

OutPut: 0 or 1

for $j \in L$ do

for $k_{i} \in S$ do

if $k_{i} \in V$ then

compute $a_{j, i, 1}=H_{1}\left(k_{j, i}, C_{j, 1}, S_{j}\right)$;

compute $a_{j, i, 2}=H_{1}\left(k_{j, i}, C_{j, 2}, V_{j}\right)$;

else

end

return error and termination of the algorithm;

end

compute $b_{j, 1}=H_{2}\left(j, C_{j, 1}, S_{j}\right)$;

compute $b_{j, 2}=H_{2}\left(j, C_{j, 2}, V_{j}\right)$;

compute LHS $=\prod_{j \in L} e\left(C_{j, 1}, g_{2}^{\sum_{k_{i} \in S_{j}} \alpha^{N+1-i} a_{j, i, 1}}\right)^{b_{j, 1}} e\left(C_{j, 2}, g_{2}^{\sum_{k_{i} \in V_{j}} \alpha^{N+1-i} a_{j, i, 2}}\right)^{b_{j, 2}}$;

compute RHS $=e \quad\left(\pi, g_{2}\right) g_{T}^{\alpha^{N+1} \sum_{j \in[L]}\left(\sum_{k_{i} \in S_{j}} m_{j, i} \cdot a_{j, i, 1} \cdot b_{1}+\sum_{k_{i} \in V_{j}} u_{j, i} \cdot a_{j, i, 2} \cdot b_{2}\right)}$

if $L E Q=R E Q$ then

output 1;

else

output 0 ;

\subsubsection{Computational costs analysis}

In this section, we analyze the calculation cost of each algorithm in this IVC. In the following table, $m$ represents the number of committed users. $b_{j}$ represents the number of proofs for aggregation operations in single commitment, and the subscript $j$ represents the number of corresponding commitments. $l$ represents the number of commitments for aggregation operations. $\left|G_{i}^{e}\right|$ represents exponentiation in the group. $|P|$ represents the bilinear pairings computation.

Table 1: Computational costs analysis

\begin{tabular}{ll}
\hline Algorithm & Time \\
\hline Insert & $\left|G_{1}^{e}\right|$ \\
Open & $2 m\left|G_{1}^{e}\right|$ \\
SCA.Agg & $2 b\left|G_{1}^{e}\right|$ \\
SCA.Ver & $\left|G_{1}^{e}\right|+2 b\left|G_{2}^{e}\right|+3|P|$ \\
MCA.Agg & $\left(2 \sum_{j} b_{j}\right)\left|G_{1}^{e}\right|$ \\
MCA.Ver & $\left|G_{1}^{e}\right|+2 \sum_{j} b_{j}\left|G_{2}^{e}\right|+(2 l+1)|P|$ \\
\hline
\end{tabular}

\subsubsection{Correctness analysis}

This section describes in detail the correctness analysis of SCA.Ver algorithm and MCA.Ver algorithm.

Correctness of SCA.Ver algorithm: 
The correctness of SCA.Ver algorithm can be proved by verifying the validity of equation (4).

The $L E Q$ can be rewritten as:

$$
\begin{aligned}
& \text { e }\left(g_{1}^{\sum_{k_{j} \in M} m_{j} \alpha^{j}}, g_{2}^{\sum_{k_{i} \in S} \alpha^{N+1-i} a_{i, 1}}\right)^{b_{1}} \mathrm{e}\left(g_{1}^{\sum_{k_{j} \in U} u_{j} \alpha^{j}}, g_{2}^{\sum_{k_{i} \in V} \alpha^{N+1-i} a_{i, 2}}\right)^{b_{2}} \\
& =\mathrm{e}\left(g_{1}, g_{2}\right)^{b_{1} \cdot\left(\sum_{k_{j} \in M} m_{j} \alpha^{j}\right)\left(\sum_{k_{i} \in S} \alpha^{N+1-i} a_{i, 1}\right)} \mathrm{e}\left(g_{1}, g_{2}\right)^{b_{2} \cdot\left(\sum_{k_{j} \in U} u_{j} \alpha^{j}\right)\left(\sum_{k_{i} \in V} \alpha^{N+1-i} a_{i, 2}\right)} \\
& =\mathrm{e}\left(g_{1}, g_{2}\right)^{b_{1} \cdot\left(\sum_{k_{j} \in M} m_{j} \alpha^{j}\right)\left(\sum_{k_{i} \in S} \alpha^{N+1-i} a_{i, 1}\right)+b_{2} \cdot\left(\sum_{k_{j} \in U} u_{j} \alpha^{j}\right)\left(\sum_{k_{i} \in V} \alpha^{N+1-i} a_{i, 2}\right)} \\
& =\mathrm{e}\left(g_{1}, g_{2}\right)^{b_{1} \cdot\left(\sum_{k_{i} \in S} a_{i, 1} \sum_{k_{j} \in M} m_{j} \alpha^{N+1-i+j}\right)+b_{2} \cdot\left(\sum_{k_{i} \in V} a_{i, 2} \sum_{k_{j} \in U} u_{j} \alpha^{N+1-i+j}\right)} .
\end{aligned}
$$

The $R E Q$ can be rewritten as:

$$
\begin{aligned}
& e\left(\pi_{S}, g_{2}\right) g_{T}^{\alpha^{N+1}\left(\sum_{k_{i} \in S} m_{i} \cdot a_{i, 1} \cdot b_{1}+\sum_{k_{i} \in V} u_{i} \cdot a_{i, 2} \cdot b_{2}\right)} \\
& =\mathrm{e}\left(\left(\prod_{k_{i} \in S} \pi_{i, 1}^{a_{i, 1}}\right)^{b_{1}} \cdot\left(\prod_{k_{i} \in V} \pi_{i, 2}^{a_{i, 2}}\right)^{b_{2}}, g_{2}\right) \cdot g_{T}^{\alpha^{N+1}\left(\sum_{k_{i} \in S} m_{i} \cdot a_{i, 1} \cdot b_{1}+\sum_{k_{i} \in V} u_{i} \cdot a_{i, 2} \cdot b_{2}\right)} \\
& =\mathrm{e}\left(\left(\prod_{k_{i} \in S}\left(g_{1}^{\sum_{k_{j} \in M, k_{j} \neq k_{i}} m_{j} \alpha^{N+1-i+j}}\right)^{a_{i, 1}}\right)^{b_{1}} \cdot\left(\prod_{k_{i} \in V}\left(g_{1}^{\sum_{k_{j} \in U, k_{j} \neq k_{i}} u_{j} \alpha^{N+1-i+j}}\right)^{a_{i, 2}}\right)^{b_{2}}, g_{2}\right) g_{T}^{\alpha^{N+1}\left(\sum_{k_{i} \in S} m_{i} \cdot a_{i, 1} \cdot b_{1}+\sum_{k_{i} \in V} u_{i} \cdot a_{i, 2} \cdot b_{2}\right)} \text {. } \\
& =\mathrm{e}\left(g_{1}^{b_{1} \sum_{k_{i} \in S} a_{i, 1} \sum_{k_{j} \in M, k_{j} \neq k_{i}} m_{j} \alpha^{N+1-i+j}} \cdot g_{1}^{b_{2} \sum_{k_{i} \in V} a_{i, 2} \sum_{k_{j} \in U, k_{j} \neq k_{i}} u_{j} \alpha^{N+1-i+j}}, g_{2}\right) \cdot g_{T}^{\alpha^{N+1}\left(\sum_{k_{i} \in S} m_{i} \cdot a_{i, 1} \cdot b_{1}+\sum_{k_{i} \in V} u_{i} \cdot a_{i, 2} \cdot b_{2}\right)} \\
& =\mathrm{e}\left(g_{1}, g_{2}\right)^{b_{1} \sum_{k_{i} \in S} a_{i, 1} \sum_{k_{j} \in M, k_{j} \neq k_{i}} m_{j} \alpha^{N+1-i+j}+b_{2} \sum_{k_{i} \in V} a_{i, 2} \sum_{k_{j} \in U, k_{j} \neq k_{i}} u_{j} \alpha^{N+1-i+j}} \\
& +\mathrm{e}\left(g_{1}, g_{2}\right)^{\alpha^{N+1+i-i}\left(\sum_{k_{i} \in S} m_{i} \cdot a_{i, 1} \cdot b_{1}+\sum_{k_{i} \in V} u_{i} \cdot a_{i, 2} \cdot b_{2}\right)}
\end{aligned}
$$

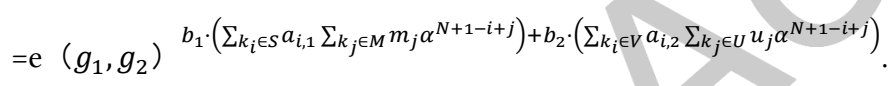

The equation holds. If the proof and commitment are generated correctly, the output of the SCA.Ver algorithm is 1 .

\section{Correctness of $\mathrm{MCA}$. Ver algorithm:}

MCA.Ver is an extended algorithm of SCA.Ver, and the proof method is similar to that of SCA.Ver.The correctness of MCA.Ver algorithm can be proved by verifying the validity of equation (6).

The aggregated proof is:

$\pi=\prod_{r \in L} \mathrm{e}\left(g_{1}, g_{2}\right)^{b_{r, 1} \sum k_{i} \in S_{r}} a_{r, i, 1} \sum_{k_{j} \in M_{r, k} \neq k_{j}} m_{r, i} \alpha^{N+1-i+j}+b_{r, 2} \sum_{k_{i} \in V_{r}} a_{r, i, 2} \sum_{k_{j} \in U_{r}, k_{j} \neq k_{i}} u_{r, i} \alpha^{N+1-i+j}$,

After substituting $\pi$ into the equation (6):

$L E Q=R E Q=e\left(g_{1}, g_{2}\right)^{\sum_{r \in L}\left(b_{r, 1} \cdot\left(\sum_{k_{i} \in S_{r}} a_{r, i, 1} \sum_{k_{j} \in M_{r}} m_{r, j} \alpha^{N+1-i+j}\right)+b_{r, 2} \cdot\left(\sum_{k_{i} \in V_{r}} a_{r, i, 2} \sum_{k_{j} \in U_{r}} u_{r, j} \alpha^{N+1-i+j}\right)\right)}$.

The equation holds. If the proof and commitment are generated correctly, the output of the MCA.Ver algorithm is 1. 


\subsection{6 security proof}

The security of the commitment scheme mainly refers to binding. If l-WBDHE assumption in the AGM (Algebraic Group Model) +ROM (Random Oracle Model) model is correct, then the scheme has binding. Suppose the adversary is trying to break the scheme, then the adversary needs to calculate commitment $C_{1}=g_{1}^{W^{T} R}, C_{2}=g_{1}^{Y^{T} R}$ (W is the set of committed values, $R$ is the set of key $k_{i}$ corresponding to $\alpha^{i}$, and $Y$ is the set of insert times.) and provide a proof $\pi_{S}$ of $(S, V, M[S], U[V])$ that can be accepted by the validation algorithm. where $W[S] \neq M[S], Y[V] \neq U[V]$ and $g_{1}^{\alpha^{N+1}}$ is unknown to the adversary. By comparing the coefficients of $g_{T}^{\alpha^{N+1}}$ on both sides of the verification algorithm, we can get

$$
W[S]^{T} A_{1} b_{1}+Y[V]^{T} A_{2} b_{2} \equiv_{p} M[S]^{T} A_{1} b_{1}+U[V]^{T} A_{2} b_{2}
$$

Where $A_{1}=\left(\left(\mathrm{H}\left(k_{i}, C_{1}, \mathrm{~S}\right), k_{i} \in S\right), A_{2}=\left(\left(\mathrm{H}\left(k_{i}, C_{2}, \mathrm{~V}\right), k_{i} \in V\right)\right.\right.$. In order to break the system, the adversary needs to build $\mathrm{W}[\mathrm{S}] \neq \mathrm{M}[\mathrm{S}], \mathrm{Y}[\mathrm{V}] \neq \mathrm{U}[\mathrm{V}]$ and

$$
W[S]^{T} A_{1} b_{1}+Y[V]^{T} A_{2} b_{2} \equiv_{p} M[S]^{T} A_{1} b_{1}+U[V]^{T} A_{2} b_{2}
$$

\section{Step1: Set up Luck-Hash query}

Since there are two hash algorithms in this scheme, two random oracle need to be constructed. First, the random oracle $H_{1}$ is set up, and the algebraic adversary can issue queries against the oracle $H_{1}$. For the query $\left(k_{i}, C, S\right)$, the oracle randomly returns $a_{i, 1} \in Z_{p}$. For the query $\left(k_{i}, C, V\right)$, the oracle randomly returns $a_{i, 2} \in Z_{p}$. Then the random oracle $H_{2}$ is set, and the algebraic adversary can issue a query against the oracle $H_{2}$. For queries $(C, S)$, the oracle randomly returns $b_{1}$. For query $(C, V)$, the oracle randomly returns $b_{2}$. Since the adversary is algebraic, it must output the set $W, X, Y, Z \in Z_{p}$ that satisfies

$$
\begin{aligned}
& C_{1}=g_{1}^{\sum_{k_{i} \in[M]} w_{i} \alpha^{i}+\sum_{k_{j} \in[M-1]} x_{j} \alpha^{N+1+j}}=g_{1}^{W^{T} \cdot R+\alpha^{N} \cdot X^{T} R[-1]} \\
& C_{2}=g_{1}^{\sum_{k_{i} \in[U]} y_{i} \alpha^{i}+\sum_{k_{j} \in[U-1]} z_{j} \alpha^{N+1+j}}=g_{1}^{Y^{T} \cdot R+\alpha^{N} \cdot Z^{T} R[-1]}
\end{aligned}
$$

If the adversary needs to win, the Luck-Hash query should satisfy $\mathrm{W}[\mathrm{S}] \neq \mathrm{M}[\mathrm{S}], \mathrm{Y}[\mathrm{V}] \neq \mathrm{U}[\mathrm{V}]$ and

$$
\left(M[S]^{T}-W[S]^{T}\right) A_{1} b_{1}+\left(U[V]^{T}-Y[V]^{T}\right) A_{2} b_{2} \equiv_{p} 0
$$

\section{Step2: Calculate $g_{1}^{\alpha^{N+1}}$}

Suppose algebra adversary to damage the system, then he will output $\left(C, S^{*}, V^{*}, M^{*}\left[S^{*}\right], U^{*}\left[V^{*}\right], \pi_{S^{*}}^{*}\right)$ satisfying $W^{*}\left[S^{*}\right] \neq M^{*}\left[S^{*}\right], Y\left[V^{*}\right] \neq U^{*}\left[V^{*}\right]$.

Since $\pi_{S}^{*}$ is an acceptable proof, we have:

$$
e\left(C_{1}, g_{2}^{\sum_{k_{i} \in S^{*}} \alpha^{N+1-i} a_{i, 1}}\right)^{b_{1}} e\left(C_{2}, g_{2}^{\sum_{k_{i} \in V^{*}} \alpha^{N+1-i} a_{i, 2}}\right)^{b_{2}}=e\left(\pi_{S^{*}}^{*}, g_{2}\right) g_{T}^{\alpha^{N+1}\left(\sum_{k_{i} \in S^{*}} m_{i} \cdot a_{i, 1} \cdot b_{1}+\sum_{k_{i} \in V^{*}} u_{i} \cdot a_{i, 2} \cdot b_{2}\right)}
$$

According to the properties of bilinear pair operation, we can rewrite equation (12) as:

$$
C_{1}^{\sum_{k_{i} \in S^{*}} \alpha^{N+1-i} a_{i, 1} \cdot b_{1}} \cdot C_{2}^{\sum_{k_{i} \in V^{*}} \alpha^{N+1-i} a_{i, 2} \cdot b_{2}}=\pi_{S^{*}}^{*} \cdot g_{1}^{\alpha^{N+1}\left(\sum_{k_{i} \in S^{*}} m_{i} \cdot a_{i, 1} \cdot b_{1}+\sum_{k_{i} \in V^{*}} u_{i} \cdot a_{i, 2} \cdot b_{2}\right)}
$$

Substitute equations (9) and (10) into equation (13), and the left side of equation (13) can be rewritten as: 


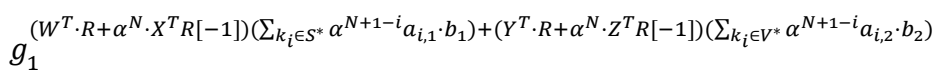

The right side of equation (13) can be rewritten as:

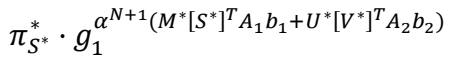

Rewrite the left side as the product of the term containing $g_{1}^{\alpha^{N+1}}$ and other terms:

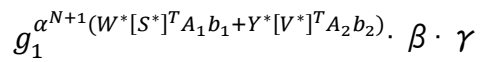

Where

$\left.\beta=g_{1}^{\alpha^{N+1-i}\left(W[-i]^{T} \cdot R[-i] \cdot a_{i, 1} b_{1}+Y[-i]^{T} \cdot R[-i] \cdot a_{i, 2} b_{2}\right)}, \gamma=g_{1}^{\alpha^{N} \cdot X^{T} R[-1]\left(\sum_{i} \in S^{*}\right.} \alpha^{N+1-i} a_{i, 1} \cdot b_{1}\right)+\alpha^{N} \cdot Z^{T} R[-1]\left(\sum_{k_{i} \in V^{*}} \alpha^{N+1-i} a_{i, 2} \cdot b_{2}\right)$

Equation (13) is rewritten as

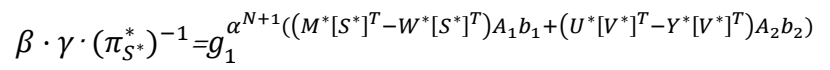

Since $W[S] \neq M[S], Y[V] \neq U[V]$, and there is no H-luck query, so we must have one

$$
\left(\left(M^{*}\left[S^{*}\right]^{T}-W^{*}\left[S^{*}\right]^{T}\right) A_{1} b_{1}+\left(U^{*}\left[V^{*}\right]^{T}-Y^{*}\left[V^{*}\right]^{T}\right) A_{2} b_{2}\right) \neq 0
$$

Then $g_{1}^{\alpha^{N+1}}$ can be calculated, and the l-WBDHE assumption is broken. In the same way, the binding of MCA.Ver can be proved.

\section{SCHEME COMPARISON}

\subsection{The Performance Comparison}

Table 2 asymptotic compares the commitment scheme of this paper with other commitment schemes. Where $n$ represents the number of committed data pieces, $b$ is a subset of committed data pieces, and $L$ is the number of commitments. The complexity in this table is asymptotic in terms of the number of exponents, pairings and field operations. Com refers to the time to calculate commitment. Insert refers to the time to insert a new element. Sub-P refers to the time to generate the proof of the data pieces of subset $b$. Sub-V refers to the time to verify the proof of the data pieces of the subset $b$. S-Agg refers to the time at which the proofs of the same commitment are aggregated. MAgg refers to the time for the aggregation of proof of multiple commitments. RP refers to whether replay attacks can be prevented. One means that only one aggregation can be performed.

Table 2: Scheme performance comparison

\begin{tabular}{llllcccc}
\hline Scheme & Com & Insert & Sub-P & Sub-V & S-Agg & M-Agg & RP \\
\hline Merkle tree & $O(n)$ & $\times$ & $O(b \log n)$ & $O(b \log n)$ & $\times$ & $\times$ & $\times$ \\
LM19-CDH [39] & $O(n)$ & $\times$ & $O(b n)$ & $O(b)$ & $\times$ & $\times$ & $\times$ \\
GRWZ20 [42] & $O(n)$ & $\times$ & $O(b n)$ & $O(b)$ & $O(b)$ & $O(L b)$ & $\times$ \\
AR20 [43] & $O(n)$ & $O(1)$ & $\times$ & $\times$ & one & $\times$ & $\sqrt{ }$ \\
This work & $O(n)$ & $O(1)$ & $O(b n)$ & $O(b)$ & $O(b)$ & $O(L b)$ & $\sqrt{ }$ \\
\hline
\end{tabular}

According to table 2, the advantage of vector commitment over Merkle tree is that it has the aggregation function of proofs. Although the computational cost of vector commitment is greater than that of Merkle tree, using the 
aggregation function of vector commitment can greatly reduce the communication cost of proofs. The scheme in this paper combines the advantages of GRWZ20 and AR20. Not only can the insertion function be realized, but also the aggregation of proofs can be realized.

\subsection{Proof Size Comparison}

Table 3 asymptotic compares the commitment scheme of this paper with other commitment schemes in terms of proof size. Where $n$ is the number of commitment data pieces, $b$ is a subset of commitment data pieces, and $L$ is the number of commitments. The complexity in this table is asymptotic in terms of the number of exponents, pairings and field operations. S-proof refers to the proof size of single data piece. B-proof refers to the Proof size of the data pieces of subset $b$. L-proof is the proof size of $L b$ data pieces. $\mathrm{PP}$ is the size of the public parameter.

Table 3 Comparison of proof size and public parameter size

\begin{tabular}{lcccc}
\hline Scheme & S-proof & B-Proof & L-Proof & PP \\
\hline Merkle tree & $O(\log n)$ & $O($ blogn $)$ & $O($ Lblogn $)$ & $\times$ \\
LM19-CDH [39] & $O(1)$ & $O(1)$ & $O(L)$ & $O\left(n^{2}\right)$ \\
GRWZ20 [42] & $O(1)$ & $O(1)$ & $O(1)$ & $O(n)$ \\
AR20 [43] & $O(1)$ & $O(b)$ & $O(L b)$ & $O(1)$ \\
This work & $O(1)$ & $O(1)$ & $O(1)$ & $O(n)$ \\
\hline
\end{tabular}

According to table 3, in contrast to Merkle trees, the proof size of a single data piece of vector commitment does not change with $n$. LM19-CDH scheme cannot realize the aggregation function of proofs among different commitments, so the L-proof of this scheme will increase with the increase of the number of commitments. The AR20 scheme can only achieve one-hop aggregation, so its B-Proof and L-proof sizes are not fixed. The B-proof and L-proof of GRWZ20 and this work are of fixed size.

The focus of this paper is to reduce the communication cost of data correctness verification. When verifying the correctness of data pieces, the main communication cost is the cost of transmitting proof. Therefore, the experimental content of this paper mainly focuses on testing the proof size of different schemes under different experimental settings. In order to show the performance of the scheme in this paper more intuitively, we compare the schemes under 128-bit security. For the quotient group, 2048 bit group is used in this paper. For the bilinear group, $B L S 12+381$ is used in this paper. We compare the schemes from three aspects: the size of a single proof, the proof size of $b$ data pieces, and the proof size of $b L$ data pieces. 


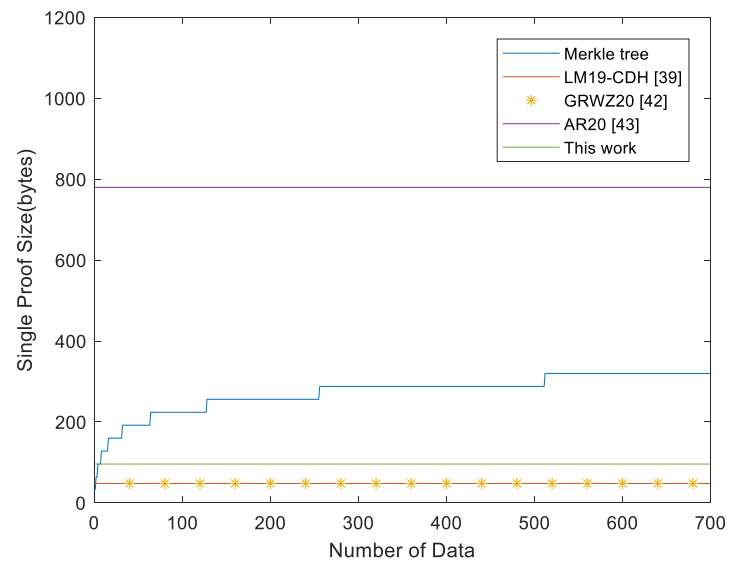

Fig. 8: Comparison of the proof size of a single data piece

First, we compare the relationship between the proof size and the number of transactions per block. LM19-CDH scheme, GRWZ20 scheme and AR20 scheme are bilinear group schemes, so we constructed the scheme according to BLS12+381 standard. AR20 is a hidden order group RSA scheme, so we use 2048-bit group. Since the proof size of the vector commitment scheme is related to the group element size, the proof size is not affected by the number of transactions per block. The proof size of the Merkle tree is affected by the number of committed data pieces, and the proof size is equal to $32 *\left(f \operatorname{loor}\left(\log _{2} N\right)+1\right)$ bytes $(N$ is number of committed data pieces). The proof size of the other schemes is fixed. Figure 8 shows the change in the proof size with the number of committed data pieces.

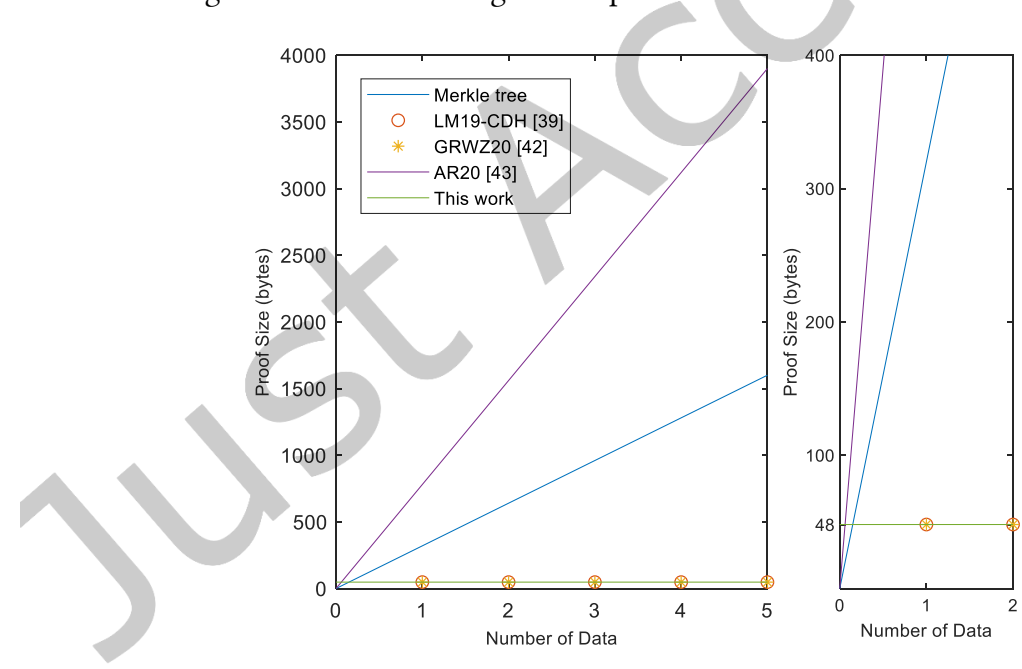

Fig. 9: Comparison of the proof size of $b$ data pieces

Second, we tested the relationship between the number of data pieces to be verified and the size of the proof. The group setup is the same as in the above experiment. In this experiment, we set each block to handle 1000 transactions, where the Merkle proof size of a single data piece is fixed. Since the Merkle tree scheme and the AR20 scheme do not have aggregation function, the size of their proof increases with the number of data pieces to be validated. $\mathrm{LM}-\mathrm{CDH}$, 
AR20 and this work have fixed-size proof. Figure 9 shows the relationship between $b$ and the proof size. The right image shows the details of the left image at points 0 to 2 .
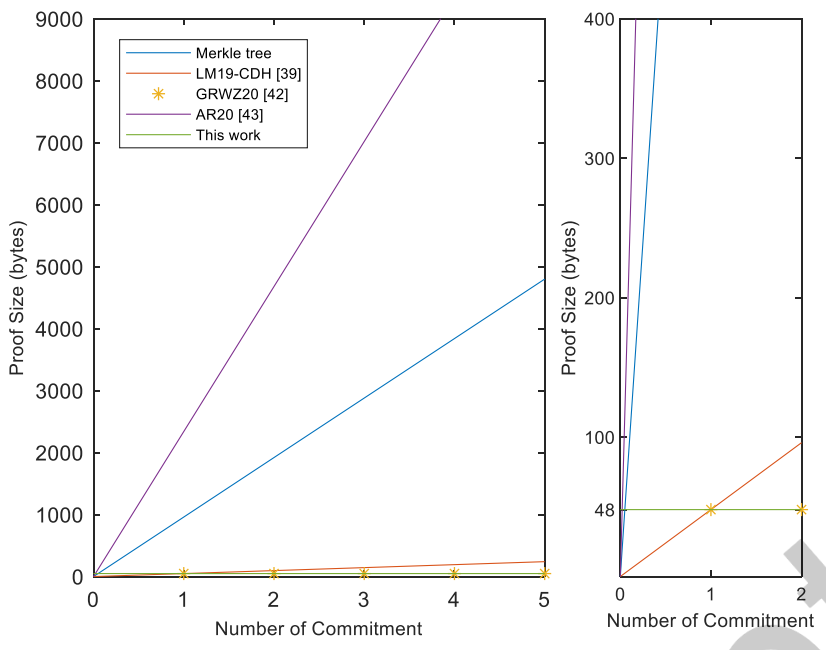

Fig. 10: Comparison of the proof size of bL data pieces

Finally, we test the relationship between the proof size and the number of commitments. The group setup is the same as in the above experiment. In this experiment, we still set the number of transactions per block to 1000 . We set each commitment have three data pieces that need to be aggregated. Merkle tree, AR20, LM-CDH are affected by $L$. The proof size of the other schemes is fixed. Figure 10 shows the relationship between $\mathrm{L}$ and the proof size. The right image shows the details of the left image at points 0 to 2 .

\section{CONCLUSION AND FUTURE WORK}

This paper uses IVC to improve the traditional blockchain. Then the S-BDS was built using the improved blockchain in zero-trust IoT. Compared to traditional blockchains that use Merkle trees to store data, our scheme not only greatly reduces the proof size of a single data piece, but also enables the aggregation of proofs between different shards. This can effectively reduce communication consumption and improve communication efficiency, so that the blockchain system can better integrate with the ZIoT system.

In the research of this paper, our blockchain system still has a limit on the number of users. The system is vulnerable to DoS attack in the process of user registration, and the attacker will continue the user registration transaction to make the system reach the user limit. A natural extension is to achieve an unlimited number of users. We make it our future work.

\section{ACKNOWLEDGMENTS}

This work was supported by the NSFC $(61772280,62072249,61772454,62072056)$. This work was funded by the Researchers Supporting Project No. (RSP-2021/102) King Saud University, Riyadh, Saudi Arabia. Yongjun Ren and Neal Xiong are the co-corresponding authors. 


\section{REFERENCES}

[1] Condry Michael W. and Catherine Blackadar Nelson. 2016. Using smart edge IoT devices for safer, rapid response with industry IoT control operations. Proceedings of the IEEE 104, 5 (2016) 938-946.

[2] Sodhro, Ali Hassan, Sandeep Pirbhulal, and Arun Kumar Sangaiah.2018. Convergence of IoT and product lifecycle management in medical health care. Future Generation Computer Systems, 86 (2018), 380-391. https://doi.org/10.1016/j.future.2018.03.052.

[3] Yongjun Ren, Jian Qi, Yaping Cheng, Jin Wang, and Osama Alfarraj. 2020. Digital Continuity Guarantee Approach of Electronic Record Based on Data Quality Theory. Computers, Materials and Continua 63, 3 (2020), 1471-1483. https://doi.org/10.32604/CMC.2020.06745.

[4] Wei Song, Ning Feng, Yifei Tian, Simon Fong, and Kyungeun Cho. 2018. A deep belief network for electricity utilisation feature analysis of air conditioners using a smart IoT platform. Journal of Information Processing Systems 14, 1 (2018), 162-175.

[5] Jin Wang, Yaqiong Yang, Tian Wang, R Simon Sherratt, Jingyu Zhang. 2020. Big data service architecture: a survey. Journal of Internet Technology, 21, 2, 393-405. https://doi.org/10.3966/160792642020032102008.

[6] Yuanyuan Zeng, Naixue Xiong, Jong Hyuk Park, and Guilin Zheng. 2010. An emergency-adaptive routing scheme for wireless sensor networks for building fire hazard monitoring. Sensors 10, 6(2010), 6128-6148. https://doi.org/10.3390/s100606128.

[7] Maliha Sultana, Afrida Hossain, Fabiha Laila, Kazi Abu Taher, Muhammad Nazrul Islam. 2020. Towards developing a secure medical image sharing system based on zero trust principles and blockchain technology. BMC Medical Informatics and Decision Making 20, 1(2020), 1-10. https://doi.org/10.1186/s12911-020-01275-y.

[8] Shanshan Zhao, Shancang Li, Fuzhong Li, Wuping Zhang, and Muddesar Iqbal. 2020. Blockchain-Enabled User Authentication in Zero Trust Internet of Things. In International Conference on Security and Privacy in New Computing Environments. Springer, Lyngby, Denmark, 265-274. https://doi.org/10.1007/978-3-030-66922-5_18.

[9] Yongjun Ren, Yan Leng, Yaping Cheng, and Jin Wang. 2019. Secure data storage based on blockchain and coding in edge computing. Mathematical Biosciences and Engineering 16, 4 (2019), 1874-1892. https://doi.org/10.3934/mbe.2019091.

[10] Suparna Dhar and Indranil Bose. 2021. Securing IoT Devices Using Zero Trust and Blockchain. Journal of Organizational Computing and Electronic Commerce 31, 1(2021), 18-34. https://doi.org/10.1080/10919392.2020.1831870.

[11] Mayra Samaniego and Ralph Deters. 2018. Zero-trust hierarchical management in iot. In 2018 IEEE international congress on Internet of Things (ICIOT). IEEE, San Francisco, CA, USA, 88-95. https://doi.org/10.1109/ICIOT.2018.00019.

[12] Mahdi Zamani, Mahnush Movahedi, and Mariana Raykova. 2018. RapidChain: Scaling Blockchain via Full Sharding. In Proceedings of the 2018 ACM SIGSAC Conference on Computer and Communications Security (CCS '18). Association for Computing Machinery, New York, NY, USA, 931-948.https://doi.org/10.1145/3243734.3243853.

[13] Qi Zhang, Chunjie Zhou, Yu-Chu Tian, Naixue Xiong, Yuanqing Qin, and Bowen Hu. 2017. A fuzzy probability Bayesian network approach for dynamic cybersecurity risk assessment in industrial control systems. IEEE Transactions on Industrial Informatics 14, 6, 2497-2506. https://doi.org/ 10.1109/TII.2017.2768998.

[14] Aniket Kate, Gregory M. Zaverucha, and Lan Goldberg. 2010. Constant-Size Commitments to Polynomials and Their Applications. In Proceedings of the ASIACRYPT 2010, 6477 LNCS:177-94. https://doi.org/10.1007/978-3-642-17373-8_11.

[15] Jin Wang, Yu Gao, Wei Liu, Arun Kumar Sangaiah, and Hye Jin Kim. 2019. An Intelligent Data Gathering Schema with Data Fusion Supported for Mobile Sink in Wireless Sensor Networks. International Journal of Distributed Sensor Networks 15, 3 (2019). https://doi.org/10.1177/1550147719839581.

[16] Zhuang Peng, Zamir Talha, and Liang Hao. 2020. Blockchain for Cyber Security in Smart Grid: A Comprehensive Survey. IEEE Tran sactions on Industrial Informatics. https://doi.org/10.1109/TII.2020.2998479.

[17] Weiwei Fang, Xuening Yao, Xiaojie Zhao, Jianwei Yin, and Naixue Xiong. 2016. A stochastic control approach to maximize profit on service provisioning for mobile cloudlet platforms. IEEE Transactions on Systems, Man, and Cybernetics: Systems 48,4(2016), 522-534. https://doi.org/10.1109/TSMC.2016.2606400.

[18] Lee Suk Kyu, Mungyu Bae, and Hwangnam Kim. 2017. Future of IoT networks: A survey. Applied Sciences7, 10 (2017), $1072-1096$.

[19] Yongjun Ren, Yan Leng, Fujian Zhu, Jin Wang, and Hye Jin Kim. 2019. Data Storage Mechanism Based on Blockchain with Privacy Protection in Wireless Body Area Network. Sensors (Switzerland) 19, 10 (2019), 1-16. https://doi.org/10.3390/s19102395.

[20] Jin Wang, Yu Gao, Chang Zhou, R. Simon Sherratt, and Lei Wang. 2020. Optimal Coverage Multi-Path Scheduling Scheme with Multiple Mobile Sinks for WSNs. Computers, Materials and Continua 62, 2 (2020), 695-711. https://doi.org/10.32604/cmc.2020.08674

[21] Ke-Ping Yu, Liang Tan, Moayad Aloqaily, Hekun Yang, and Yaser Jararweh. 2021. Blockchain-enhanced data sharing with traceable and direct revocation in IIoT. IEEE transactions on industrial informatics 17, 11 (2021), 7669-7678. https://doi.org/10.1109/TII.2021.3049141.

[22] Contreras-Castillo Juan, Sherali Zeadally, and Juan Antonio Guerrero-Ibañez. 2017. Internet of vehicles: architecture, protocols, and security. IEEE internet of things Journal 5, 5 (2017), 3701-3709.

[23] Mou Wu, Liansheng Tan, and Naixue Xiong. 2015. A structure fidelity approach for big data collection in wireless sensor netwo rks. Sensors 15, 1(2015), 248-273. https://doi.org/10.3390/s150100248.

[24] Baotong Chen, Jiafu Wan, Lei Shu, Peng Li, Mithun Mukherjee, And Boxing Yin. 2017. Smart factory of industry 4.0: Key technologies, application case, and challenges. IEEE Access2017,6 (2017), 6505-6519. https://doi.org/10.1109/ACCESS.2017.2783682.

[25] Yongjun Ren, Yan Leng, Jian Qi, Pradip Kumar Sharma, Jin Wang, Zafer Almakhadmeh, and Amr Tolba. 2019. Multiple Cloud Storage Mechanism Based on Blockchain in Smart Homes. Future Generation Computer Systems 115 (2021), 304-13. https://doi.org/10.1016/j.future.2020.09.019.

[26] Haochen Pan, Xuheng Duan, yingjian Wu, Lewis Tseng, Moayad Aloqaily, and Boukerche. 2020. BBB: A Lightweight Approach to Evaluate 
Private Blockchains in Clouds. In GLOBECOM 2020-2020 IEEE Global Communications Conference. IEEE, Taipei, Taiwan, 1-6. https://10.1109/GLOBECOM42002.2020.9322354.

[27] Jin Wang, Yu Gao, Xiang Yin, Feng Li, and Hye Jin Kim. 2018. An Enhanced PEGASIS Algorithm with Mobile Sink Support for Wireless Sensor Networks. Wireless Communications and Mobile Computing (2018). https://doi.org/10.1155/2018/9472075.

[28] Liang Tan, Huan Xiao, Keping Yu, Moayad Aloqaily, and Yaser Jararweh. 2021. A blockchain-empowered crowdsourcing system for 5g-enabled smart cities. Computer Standards \& Interfaces 76, 103517. https://doi.org/10.1016/j.csi.2021.103517.

[29] Hao Wang, Shenglan Ma, Chaonian Guo, Yulei Wu, Hong-Ning Dai, and Di Wu. 2021. Blockchain-Based Power Energy Trading Management. ACM Trans. Internet Technol. 21, 2, Article 43 (March 2021), 16 pages. https://doi.org/10.1145/3409771.

[30] Lewis Tseng, Xinyu Yao, Safa Otoum, Moayad Aloqaily, and Yaser Jararweh. 2020. Blockchain-based database in an IoT environment: challenges, opportunities, and analysis. Cluster Computing 23, 3 (2020), 2151-2165. https://doi.org/10.1007/s10586-020-03138-7.

[31] Yongjun Ren, Fujian Zhu, Pradip Kumar Sharma, Tian Wang, Jin Wang, Osama Alfarraj, and Amr Tolba. 2020. Data Query Mechanism Based on Hash Computing Power of Blockchain in Internet of Things. Sensors (Switzerland) 20, 1 (2020). https://doi.org/10.3390/s20010207.

[32] Faiza Loukil, Chirine Ghedira-Guegan, Khouloud Boukadi, Aïcha-Nabila Benharkat, and Elhadj Benkhelifa. 2021. Data Privacy Based on IoT Device Behavior Control Using Blockchain. ACM Trans. Internet Technol. 21, 1, Article 23 (February 2021), 20 pages. https://doi.org/10.1145/3434776.

[33] Jin Wang, Yu Gao, Wei Liu, Wenbing Wu, and Se-jung Lim. 2019. An Asynchronous Clustering and Mobile Data Gathering Schema Based on Timer Mechanism in Wireless Sensor Networks. Computers, Materials \& Continua 58, 3 (2019), 711-725. https://doi.org/10.32604/cmc.2019.05450.

[34] Damgård Ivan and Eiichiro Fujisaki. 2002. A statistically-hiding integer commitment scheme based on groups with hidden order. International Conference on the Theory and Application of Cryptology and Information Security. Springer, Berlin, BL, Germany, 125-142 . https://doi.org/10.1007/3-540-36178-2_8.

[35] Yanzhen Qu and Naixue Xiong. 2012. RFH: A resilient, fault-tolerant and high-efficient replication algorithm for distributed cloud storage. In 2012 41st International Conference on Parallel Processing. IEEE, Pittsburgh, PA, USA, 520-529. https://doi.org/10.1109/ICPP.2012.3.

[36] Dario Catalano, Dario Fiore, and Mariagrazia Messina. 2008. Zero-Knowledge Sets with Short Proofs. In: Smart N. (eds) Advances in Cryptology. Springer, Berlin, BL, Germany, 433-450. https://doi.org/10.1007/978-3-540-78967-3_25.

[37] Benoît Libert and Moti Yung. 2010. Concise mercurial vector commitments and independent zero-knowledge sets with short proofs. In Theory of Cryptography Conference. Springer, Berlin, BL, Germany, 499-517. https://doi.org/10.1007/978-3-642-11799-2_30.

[38] Dario Catalano and Dario Fiore. 2013. Vector commitments and their applications. In 16th International Conference on Practice and Theory in Public-Key Cryptography. Springer, Berlin, BL, Germany, 55-72. https://doi.org/10.1007/978-3-642-36362-7_5.

[39] Russell W. F. Lai and Giulio Malavolta. 2019. Subvector commitments with application to succinct arguments. In 39th Annual International Cryptology Conference. Springer, Berlin, BL, Germany, 530-560. https://doi.org/10.1007/978-3-030-26948-7_19.

[40] Dan Boneh, Benedikt B"unz, and Ben Fisch. 2019. Batching techniques for accumulators with applications to iops and stateless blockchains. In 39th Annual International Cryptology Conference. Springer, Berlin, BL, Germany. https://doi.org/10.1007/978-3-030-26948-7_20.

[41] Alin Tomescu, Ittai Abraham, Vitalik Buterin, Justin Drake, Dankrad Feist, and Dmitry Khovratovich. 2020. Aggregatable subvector commitments for stateless cryptocurrencies. International Conference on Security and Cryptography for Networks. Springer, Berlin, BL, Germany, 45-64. https://doi.org/10.1007/978-3-030-57990-6_3.

[42] Sergey Gorbunov, Leonid Reyzin, Hoeteck Wee, and Zhenfei Zhang. 2020. Pointproofs: Aggregating Proofs for Multiple Vector Commitments. In Proceedings of the 2020 ACM SIGSAC Conference on Computer and Communications Security (CCS '20). Association for Computing Machinery, New York, NY, USA, 2007-2023. https://doi.org/10.1145/3372297.3417244.

[43] Shashank Agrawal and Srinivasan Raghuraman. 2020. KVaC: Key-Value Commitments for Blockchains and Beyond. International Conference on the Theory and Application of Cryptology and Information Security. Springer, Berlin, BL, Germany, 839-869.

[44] Rainer Böhme, Nicolas Christin, Benjamin Edelman, Tyler Moore. 2015. Bitcoin: Economics, technology, and governance. Journal of economic Perspectives 29, 2 (2015), 213-38. https://doi.org/10.1257/jep.29.2.213.

[45] Ruhan He, Naixue Xiong, Laurence T Yang, Jong Hyuk Park. 2011. Using multi-modal semantic association rules to fuse keywords and visual features automatically for web image retrieval. Information Fusion, 12, 3, 223-230. https://doi.org/10.1016/j.inffus.2010.02.001.

[46] Jin Wang, Xiujian Gu, Wei Liu, Arun Kumar Sangaiah, and Hye Jin Kim. 2019. An Empower Hamilton Loop Based Data Collection Algorithm with Mobile Agent for WSNs. Human-Centric Computing and Information Sciences 9, 1 (2019). https://doi.org/10.1186/s13673-019-0179-4.

[47] Ana Reyna, Cristian Martín, Jaime Chen, Enrique Soler, and Manuel Díaz. 2018. On blockchain and its integration with IoT. Challenges and opportunities. Future generation computer systems 2018, 88 (2018), 173-190. https://doi.org/10.1016/j.future.2018.05.046.

[48] Yongjun Ren, Yepeng Liu, Sai Ji, Arun Kumar Sangaiah, Jin Wang. 2018. Incentive Mechanism of Data Storage Based on Blockchain for Wireless Sensor Networks. Mobile Information Systems, (2018). https://doi.org/10.1155/2018/6874158.

[49] Zheng Wan, Naixue Xiong, Nasir Ghani, Athanasios V Vasilakos, Liang Zhou. 2014. Adaptive unequal protection for wireless video transmission over IEEE 802.11 e networks. Multimedia Tools and Applications, 72, 1, 541-571. https://doi.org/10.1007/s11042-013-1378-z.

[50] Gang Wang, Zhijie Jerry Shi, Mark Nixon, and Song Han. 2019. SoK: Sharding on Blockchain. In Proceedings of the 1st ACM Conference on Advances in Financial Technologies (AFT '19). Association for Computing Machinery, New York, NY, USA, 41-61. https://doi.org/10.1145/3318041.3355457.

[51] Jin Wang, Chenchen Han, Xiaofeng Yu, Yongjun Ren, R Simon Sherratt. 2022. Distributed Secure Storage Scheme Based on Sharding Blockchain. CMC-Computers, Materials \& Continua, 70, 3 (2022), 4485-4502. 
[52] Xiaoqin Feng, Jianfeng Ma, Yinbin Miao, Qian Meng, Ximeng Liu, Qi Jiang, and Hui Li. 2019. Pruneable sharding-based blockchain protocol. Peerto-Peer Networking and Applications 12, 4 (2019), 934-950. https://doi.org/10.1007/s12083-018-0685-6.

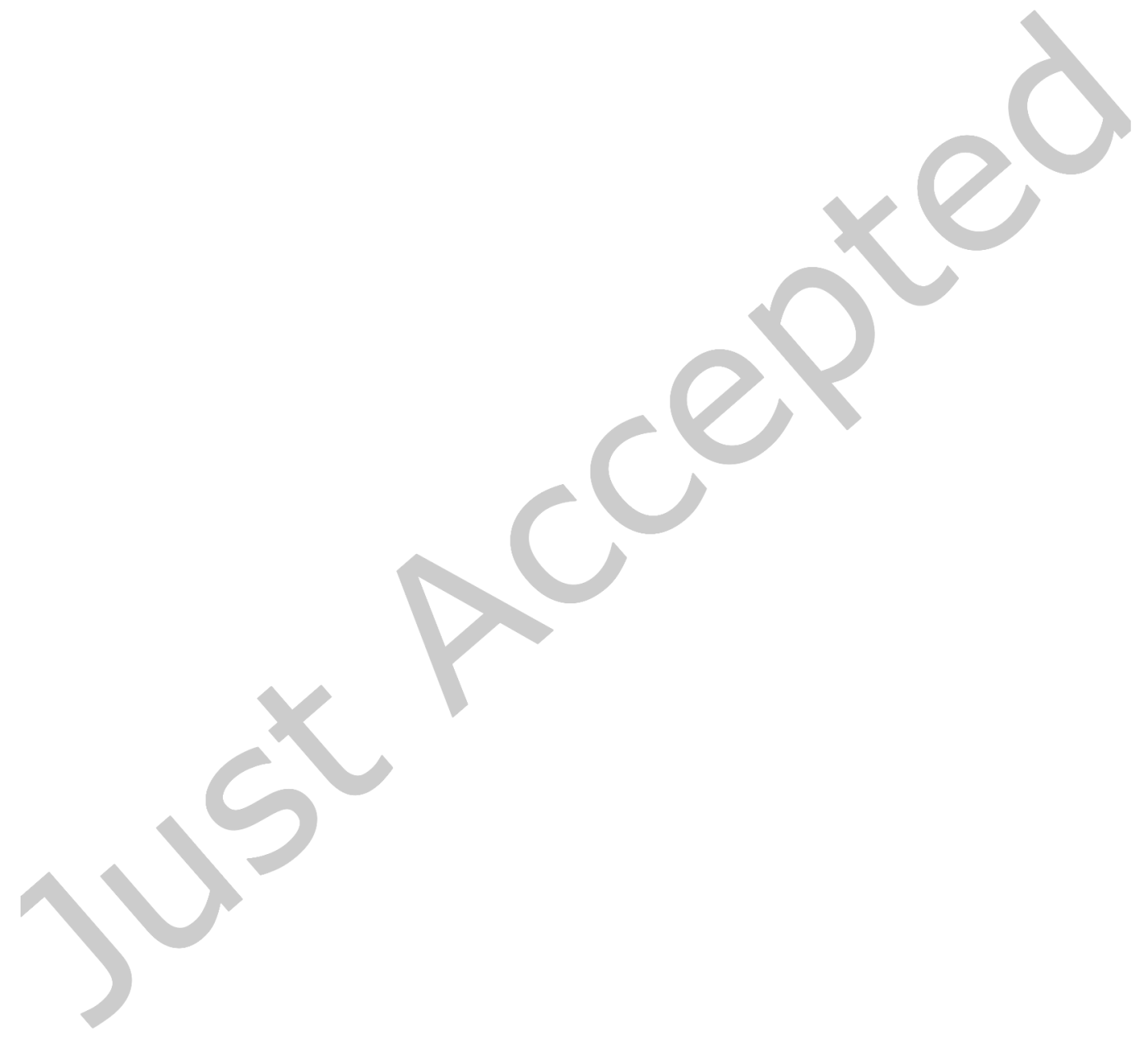

